\title{
Logics for Belief as Maximally Plausible Possibility
}

\author{
Giacomo Bonanno* \\ Department of Economics, University of California, Davis, USA \\ gfbonanno@ucdavis.edu
}

June, 2018. Revised, May 2019.

\begin{abstract}
We consider a basic logic with two primitive uni-modal operators: one for certainty and the other for plausibility. The former is assumed to be a normal operator (corresponding - semantically - to a binary Kripke relation), while the latter is merely a classical operator (corresponding - semantically - to a neighborhood structure). We then define belief, interpreted as "maximally plausible possibility", in terms of these two notions: the agent believes $\phi$ if (1) she cannot rule out $\phi$ (that is, it is not the case that she is certain that $\neg \phi)$, (2) she judges $\phi$ to be plausible and (3) she does not judge $\neg \phi$ to be plausible. We consider four interaction properties between certainty and plausibility and study how these properties translate into properties of belief (e.g. positive and negative introspection and their converses). We then prove that all the logics considered are minimal logics for the highlighted theorems. We also consider a number of possible interpretations of plausibility, identify the corresponding logics and show that some notions considered in the literature are special cases of our framework.
\end{abstract}

*This research supported by a grant from the University of California, Davis. 


\section{Introduction}

There is a large body of literature in philosophy devoted to investigating the notions of knowledge and belief. One strand in the literature takes belief as primitive and addresses the question "what is needed in order for belief to constitute knowledge?". ${ }^{1}$ Another strand takes knowledge as primitive and defines belief in terms of knowledge. ${ }^{2}$ Yet another strand in the literature takes both knowledge and belief as primitive notions and investigates the interaction between the two: for example, whether it is reasonable to postulate that if the individual believes $\phi$ then she should believe that she knows $\phi .^{3}$

Another body of literature (mainly in modal logic, computer science and game theory) takes belief as primitive but distinguishes two different, yet coexisting, types of belief, one often referred to as knowledge and the other as belief. ${ }^{4}$ Knowledge is interpreted as a stronger doxastic attitude, for example reflecting strong evidence, while belief represents a weaker doxastic attitude, reflecting an assessment of likelihood or plausibility. Some of this literature suggests that the difference between the two notions reflects a distinction between hard information and soft information. For example, van Benthem writes (van Benthem 2007, p.2):

"[...] hard information, [...] changes what I know. If I see that the Ace of Spades is played on the table, I come to know that no one holds it any more. [...] Soft information, [...] affects my beliefs without affecting my knowledge about the cards. I see you smile. This makes it more likely that you hold a trump card, but it does not rule out that you have not got one."

The distinction between hard information (giving rise to knowledge) and soft information (giving rise to belief) has been investigated within the context of belief revision. ${ }^{5}$

In this paper we start with two primitive notions and obtain from them belief as a derived notion.

\footnotetext{
${ }^{1}$ See, for example, Gettier (1963), Lehrer and Paxson (1969), Swain (1974), Williamson (2000).

${ }^{2}$ For example, belief has been defined as the epistemic possibility of knowledge: Lenzen (1978), Stalnaker (2006).

${ }^{3}$ See, for example, Hintikka (1962), Klein et al. (2018), Lenzen (1978; 2004), Stalnaker (2006).

${ }^{4}$ See, for example, Aumann (1976; 1999), Balbiani et al. (2018), Battigalli and Bonanno (1999), Friedman and Halpern (1997), van der Hoek and Meyer (1995), Kraus and Lehmann (1988), Voorbraak (1992).

${ }^{5}$ See, for example Baltag and Smets (2008), van Ditmarsch et al. (2007), van Benthem (2007; 2011) and the overview in Fiutek (2013).
} 
The first primitive notion corresponds to what is usually taken to be knowledge, but we replace it with certainty: for the sake of generality, we do not impose any of the properties that are normally associated with knowledge (e.g. positive and/or negative introspection, truth), although those properties can be added if deemed appropriate; indeed, all our results remain true if one adds to the logic of certainty the axioms that are normally used to define knowledge. ${ }^{6}$

We call our second primitive notion plausibility, representing a judgment of credibility (alternative possible interpretations of 'plausibility' are discussed in Section 6). Thus, to borrow van Benthem's example quoted above, I can be certain that no one holds the Ace of Spades (because I see it on the table) and consider it plausible that you hold a trump card (because you are grinning); furthermore, this latter judgment can co-exist with the judgment that it is also plausible that you do not hold a trump card (because I know that you are good at faking facial expressions), that is, both $\phi$ and $\neg \phi$ can, in principle, be considered plausible. Plausibility differs from possibility. The latter relates to certainty: one considers $\phi$ possible if one is not certain that $\neg \phi$ is the case. One might consider $\phi$ possible and, at the same time, not plausible or not credible. ${ }^{7}$

From the two notions of certainty and plausibility we obtain belief as a derived notion, by defining belief as maximally plausible possibility, in the sense that the agent believes that $\phi$ if and only if:

1. she cannot rule out $\phi$, that is, it is not the case that she is certain that $\neg \phi$,

2. she judges $\phi$ to be plausible, and

3. she does not judge $\neg \phi$ to be plausible.

We use the expression 'maximally plausible possibility' rather than 'plausible possibility' because of Point 3: if $\phi$ is plausible, but so is $\neg \phi$, then - according to our definition - it is not the case that the agent believes $\phi$.

For example, consider Ann who - recognising her lack of expertise-decides to base her opinions (concerning some relevant matters) on the beliefs of $n$

6 Thus we steer clear of the controversies concerning what is appropriate to postulate for knowledge. For example, Hintikka (Hintikka (1962)) and Lenzen (Lenzen (2004)) argue against Negative Introspection (if I don't know that $\phi$ then I know that I don't know) as a justifiable property of knowledge, Williamson (Williamson (2000)) offers some arguments against Positive Introspection (if I know that $\phi$ then I know that I know); on the other hand, Truth (if I know that $\phi$ then $\phi$ is indeed true) adds an external criterion, which seems indispensable for knowledge but problematic if one is merely interested in modeling the doxastic state of an individual

${ }^{7} \mathrm{~A}$ natural axiom to postulate is that plausibility implies possibility: see Axiom $\left(\mathrm{CP}_{2}\right)$ in Section 3. 
experts $(n \geq 2):{ }^{8}$ she declares herself to be certain of $p$ if all the experts believe $p$ and - since she considers all experts to be competent and reliable - she considers $p$ to be plausible if and only if at least $m$ experts believe $p$, for some fixed $1 \leq m \leq$ $n$. For simplicity, consider the case where the experts are "opinionated" (that is, each of them either believes $p$ or believes $\neg p$, for every relevant proposition $p$ ). Let $b$ be the number of experts who believe $p$ (so that, given our assumption, the remaining $n-b$ experts believe $\neg p$ ). Then $p$ is maximally plausible for Ann if and only if (1) $b \geq m$ and (2) $n-b<m$ (which is equivalent to $b \geq n-m+1$ ), that is, if and only if $b \geq \max \{m, n-m+1\}$. Thus we can distinguish three cases:

1. If $m=1$ then belief coincides with certainty: Ann believes $p$ if and only if all the experts believe $p$.

2. If $1<m<\frac{n+1}{2}$ (so that $\max \{m, n-m+1\}=n-m+1$ ) then belief differs from both plausibility and certainty: Ann believes $p$ if and only if $b \geq n-m+1$. $^{9}$

3. If $m \geq \frac{n+1}{2}$ (so that $\max \{m, n-m+1\}=m$ ) then belief coincides with plausibility: Ann believes $p$ if and only if she considers $p$ plausible. ${ }^{10}$

The logic that we develop can accommodate epistemic/doxastic states such as the one expressed in the statement "I believe, but am not certain, that Palestine is not a party to the statute of the International Court of Justice" (Beddor and Goldstein (2018))..$^{11}$.

Our approach thus falls within the literature that takes belief not as a primitive but as a derived notion. ${ }^{12}$

We start with a basic modal logic, denoted by $\mathbb{L}$, containing a certainty operator $C$, which is assumed to be a normal operator (represented, semantically,

\footnotetext{
${ }^{8}$ We assume that the experts hold consistent beliefs, that is none of the experts simultaneously believes a proposition and its negation.

${ }^{9}$ For example, if $n=12$ and $m=3$ then if $b=4$ Ann considers $p$ plausible but does not believe $p$ (because $\neg p$ is also plausible), while if $b=10$ then Ann believes $p$ although she is not certain that $p$.

${ }^{10}$ If the experts are not opinionated, let $b$ be the number of experts that believe $p, b_{\neg}$ the number of experts that believe $\neg p$ and $b_{\text {? }}$ the number of experts that neither believe $p$ nor believe $\neg p$ (thus $\left.b+b_{\neg}+b_{\text {? }}=n\right)$. Fix an $m$ such that $1 \leq m \leq n$. Then $p$ is maximally plausible if and only if $b \geq \max \left\{m, n-m-b_{\text {? }}+1\right\}$.

${ }^{11}$ Lenzen (Lenzen 2004, p.969) remarks that "from a pragmatic point of view when person $a$ says 'I believe that $p^{\prime}$, she thereby expresses that she is not convinced that $p$. "

${ }^{12}$ In the literature where belief is derived from knowledge and plausibility, one postulates either a preference/plausibility ordering over possible worlds (and then $\phi$ is defined to be believed if it is known to be true in the most preferred - or most plausible - worlds) or a plausibility measure over events (and then an individual is said to believe $\phi$ if and only if she knows that $\phi$ is more plausible than $\neg \phi$ ): see, for example, Lamarre and Shoham (1994), Moses and Shoham (1993), Friedman and Halpern (1997).
} 
by a binary relation on the set of possible world), and a plausibility operator $P$, which is assumed to be a classical operator (represented by a neighborhood structure). ${ }^{13}$ To begin with, we impose no restrictions at all on the logic of certainty and plausibility. We define the belief operator as follows:

$$
B \phi \leftrightarrow(\neg C \neg \phi \wedge P \phi \wedge \neg P \neg \phi) .
$$

First we show that it is a theorem of this basic logic that belief satisfies consistency, that is, it cannot be the case that the agent believes $\phi$ and also $\neg \phi$ (Proposition 1):

$$
B \phi \rightarrow \neg B \neg \phi .
$$

Then we consider minimal extensions of logic $\mathbb{L}$ that yield as theorems various properties that have been discussed in the literature concerning:

- the interaction of certainty and belief,

- properties of belief, such as positive introspection (if the agent believes $\phi$ then she believes that she believes $\phi$ ), negative introspection (if the agent does not believe $\phi$ then she believes that she does not believe $\phi$ ) and their converses.

These extensions of logic $\mathbb{L}$ are obtained by adding axioms concerning the interaction of certainty and plausibility. First of all, it seems contradictory to state "I find $\phi$ to be implausible (or unlikely) and yet I am certain (I know) that $\phi^{\prime \prime}$; the first axiom rules this out by requiring certainty to imply plausibility:

$$
C \phi \rightarrow P \phi \text {. }
$$

Secondly, it also seems contradictory to claim 'I find $\neg \phi$ to be plausible (or likely) and yet I am certain (I know) that $\phi^{\prime \prime}$; the second axiom rules this out by requiring certainty of $\phi$ to imply implausibility of $\neg \phi$ :

$$
C \phi \rightarrow \neg P \neg \phi .
$$

Adding Axioms $\left(C P_{1}\right)$ and $\left(C P_{2}\right)$ to the basic logic $\mathbb{L}$ yields (Proposition 5):

$$
C \phi \rightarrow B \phi \quad \text { (Certainty implies belief). }
$$

\footnotetext{
${ }^{13}$ The reasons for not assuming that the plausibility operator is a normal operator are discussed below.
} 
We then add two more interaction axioms that can be viewed as "introspection" properties, namely that if the agent judges $\phi$ to be (im)plausible than she is certain of her judgment:

$$
\begin{aligned}
\neg P \phi & \rightarrow C \neg P \phi \\
P \phi & \rightarrow C P \phi .
\end{aligned}
$$

When all four axioms $\left(C P_{1}\right)-\left(C P_{4}\right)$ are added to the basic logic $\mathbb{L}$, the following become theorems of the corresponding logic (Propositions 6-10):

$$
\begin{array}{ll}
B \phi \rightarrow C B \phi & \text { (Belief implies certainty of belief) } \\
\neg B \phi \rightarrow C \neg B \phi & \text { (Absence of belief implies certainty of absence of belief) } \\
B \phi \rightarrow B B \phi & \text { (Positive Introspection of belief) } \\
B B \phi \rightarrow B \phi & \text { (Converse of positive introspection) } \\
\neg B \phi \rightarrow B \neg B \phi & \text { (Negative Introspection of belief) } \\
B \neg B \phi \rightarrow \neg B \phi & \text { (Converse of negative introspection). }
\end{array}
$$

It is worth noting that, besides the interaction axioms $\left(C P_{1}\right)-\left(C P_{4}\right)$, no properties need to be imposed on the logic of certainty or on the logic of plausibility in order to obtain the above theorems. All of the above is shown in Sections 2 and 3.

In Section 4 we develop a possible world semantics for the logics considered in the previous sections and provide modal correspondence results, as well as results concerning the minimality of the logics considered in Section 3.

This brings us to the issue of why we model plausibility as a classical, rather than a normal, operator. Our purpose is to explore a very general logic of (a derived notion of) belief, that does not impose at the outset constraints such as the conjunction properties, according to which belief of $\phi$ and belief of $\psi$ implies belief of $(\phi \wedge \psi)$ and vice versa. For example, we want our notion of belief to be flexible enough to accommodate phenomena such as the Lottery Paradox (Kyburg $(1961 ; 1983)$ ). In 1961 Kyburg pointed out that there are circumstances where one can reasonably believe each of $n$ propositions $(n \geq 2)$ while at the same time believing the negation of their conjunction. For example, given 100 Million lottery tickets and exactly one winner, the probability of the proposition "Ticket $n$ is not the winner" (denote it by $p_{n}$ ) is virtually 1 and thus one might be disposed to believe it; on the other hand, since there is a winner, the conjunction $\left(p_{1} \wedge \cdots \wedge p_{n}\right)$ must be false and thus one might also be disposed to believe $\neg\left(p_{1} \wedge \cdots \wedge p_{n}\right)$. Kyburg argues for tolerating such 
joint inconsistency of beliefs. In the strongest logic mentioned above, namely $\mathbb{L}+\left\{C P_{1}, C P_{2}, C P_{3}, C P_{4}\right\}$, formulas of the form $B(\phi \wedge \psi) \wedge \neg(B \phi \wedge B \psi)$ as well as $B \phi \wedge B \psi \wedge \neg C \neg(\phi \wedge \psi) \wedge \neg B(\phi \wedge \psi)$ are satisfiable and thus the conjunction properties of belief do not hold (this remains the case even if one imposes the S5 logic on the certainty operator, that is, if one interprets certainty as knowledge). In Section 5 we identify further axioms that can be used to extend the basic logic $\mathbb{L}$ in such a way that the conjunction properties of belief become theorems.

In Section 6 we consider several possible interpretations of the notion of plausibility (such as plausibility as positive probability, plausibility as truth at most preferred world(s), etc.). We identify the extensions of our basic logic $\mathbb{L}$ that are implied by such interpretations and show that our framework is sufficiently flexible to accommodate various notions proposed in the literature.

In Section 7 we consider alternative minimal extensions of the basic logic $\mathbb{L}$ that yield some of the schemata considered in Section 3. Section 8 concludes. The proofs of minimality of the logics considered are given in the Appendix.

It should be noted that the results proved in this paper do not present technical challenges and are quite straightforward applications of known methods. We view the contribution of this paper as threefold:

- we provide a stripped-down logic for exploring the notion of belief as derived from the primitive notions of certainty and plausibility: the less structure one imposes, the clearer it is to grasp what is really necessary in order to obtain "desirable" properties of belief (such as positive and negative introspection),

- we point out that properties of beliefs that are normally postulated in the literature (such as positive and negative introspection) can be understood in terms of the interaction of certainty and plausibility, and

- we show that the proposed logic can accommodate various notions of belief proposed in the literature.

Our view is that, instead of seeking "the philosophically correct" notion of belief, one can rely on a flexible framework that can be restricted in several ways (with appropriate axioms) depending on the context and application one has in mind. Thus, rather than defending any particular axiom or set of axioms as "reasonable", we limit ourselves to investigating what each set of axioms (involving the primitive notions of certainty and plausibility) implies in terms of properties of the proposed notion of belief. 


\section{The basic logic}

We consider a modal logic with two modal operators: $C$ interpreted as "certainty" and $P$ interpreted as "plausibility". Thus $C \phi$ means that the individual in question, from now on called "the agent", is certain that $\phi$, and $P \phi$ means that the agent judges $\phi$ to be plausible. $C$ will be taken to be a normal operator, while we will impose no restrictions on the operator $P$ (besides assuming that is a classical operator).

The formal language is built in the usual way from a countable set of propositional variables (or atoms) At, the connectives $\neg$ (for "not") and $\vee$ (for "or") and the modal operators. ${ }^{14}$ Thus the set $\Phi$ of formulas is defined inductively as follows: $q \in \Phi$ for every atomic proposition $q \in$ At, and if $\phi, \psi \in \Phi$ then all of the following belong to $\Phi: \neg \phi,(\phi \vee \psi), C \phi$ and $P \phi$. We will follow the standard rules for omission of the parentheses.

We denote by $\mathbb{L}_{0}$ the logic determined by the following axioms and rules of inference. ${ }^{15}$

\section{AXIOMS:}

1. All propositional tautologies.

2. Axiom $K$ for $C$ :

$$
C(\phi \rightarrow \psi) \rightarrow(C \phi \rightarrow C \psi)
$$

\section{RULES OF INFERENCE:}

1. Modus Ponens:

$$
\frac{\phi, \phi \rightarrow \psi}{\psi}
$$

2. Necessitation for $C$ :

$$
\frac{\phi}{C \phi}
$$

3. Rule RE for $P$ :

$$
\frac{\phi \leftrightarrow \psi}{P \phi \leftrightarrow P \psi}
$$

\footnotetext{
${ }^{14}$ See, for example, Chellas (1984), Blackburn et al. (2001). The connectives $\wedge$ (for "and"), $\rightarrow$ (for "if ... then ...") and $\leftrightarrow$ (for "if and only if") are defined as usual: $\phi \wedge \psi=\neg(\neg \phi \vee \neg \psi), \phi \rightarrow \psi=\neg \phi \vee \psi$ and $\phi \leftrightarrow \psi=(\phi \rightarrow \psi) \wedge(\psi \rightarrow \phi)$.

${ }^{15}$ Throughout the paper, the naming of axioms and rules of inference follows Chellas (1984).
} 
Remark 1 (Derived rules of inference). It is well-known that from $K_{C},(M P)$ and $\left(\mathrm{Nec}_{\mathrm{C}}\right)$ one can derive the following rules of inference:

$$
\begin{gathered}
\frac{\phi \rightarrow \psi}{C \phi \rightarrow C \psi} \\
\frac{\phi \leftrightarrow \psi}{C \phi \leftrightarrow C \psi} \\
\frac{\phi \rightarrow \psi}{\neg C \neg \phi \rightarrow \neg C \neg \psi} \\
\phi \leftrightarrow \psi \\
\frac{\neg C \neg \phi \leftrightarrow \neg C \neg \psi}{}
\end{gathered}
$$

Remark 2 (Normality of C). It is also well-known that the following are theorems of the basic logic $\mathbb{L}_{0}$ :

$$
\begin{array}{cl}
\left(M_{C}\right) & C(\phi \wedge \psi) \rightarrow(C \phi \wedge C \psi) \\
\left(C_{C}\right) & (C \phi \wedge C \psi) \rightarrow C(\phi \wedge \psi) \\
\left(M_{C}^{\text {dual }}\right) & \neg C \neg(\phi \vee \psi) \rightarrow(\neg C \neg \phi \vee \neg C \neg \psi) \\
\left(C_{C}^{\text {dual }}\right) & (\neg C \neg \phi \vee \neg C \neg \psi) \rightarrow \neg C \neg(\phi \vee \psi)
\end{array}
$$

Remark 3. In virtue of rule $R_{K}^{C}$, the following is a theorem of logic $\mathbb{L}_{0}:{ }^{16}$

$$
\neg C \neg\left(\phi_{1} \wedge \cdots \wedge \phi_{n}\right) \rightarrow\left(\neg C \neg \phi_{1} \wedge \cdots \wedge \neg C \neg \phi_{n}\right)
$$

\footnotetext{
${ }^{16}$ Proof. For every $i=1, \ldots, n$, since $\neg \phi_{i} \rightarrow \neg\left(\phi_{1} \wedge \cdots \wedge \phi_{n}\right)$ is a tautology, by $\mathrm{R}_{\mathrm{K}}^{C}$ we get that $C \neg \phi_{i} \rightarrow C \neg\left(\phi_{1} \wedge \cdots \wedge \phi_{n}\right)$ is a theorem. Thus, by propositional logic (PL), we get that $\neg C \neg\left(\phi_{1} \wedge \cdots \wedge \phi_{n}\right) \rightarrow \neg C \neg \phi_{i}$, from which we obtain (again, by PL) that $\neg C \neg\left(\phi_{1} \wedge \cdots \wedge \phi_{n}\right) \rightarrow$ $\left(\neg C \neg \phi_{1} \wedge \cdots \wedge \neg C \neg \phi_{n}\right)$.
} 
We now extend the logic $\mathbb{L}_{0}$ by adding a third operator, namely the belief operator $B$, which is derived from $C$ and $P$. The interpretation of $B \phi$ is "the agent believes that $\phi$ ". Belief is defined as "maximally plausible possibility" in the sense that the agent believes that $\phi$ if and only if:

1. she cannot rule out $\phi$, that is, it is not the case that she is certain that $\neg \phi$,

2. she judges $\phi$ to be plausible, and

3. she does not judge $\neg \phi$ to be plausible.

Definition 2.1. The operator $B$ is defined as follows:

$$
B \phi \leftrightarrow(\neg C \neg \phi \wedge P \phi \wedge \neg P \neg \phi)
$$

We denote by $\mathbb{L}$ the logic obtained by extending the syntax of $\mathbb{L}_{0}$ to include formulas of the form $B \phi$ and by adding axiom (DefB) to $\mathbb{L}_{0}$.

First we show that, without imposing any further axioms, we obtain consistency of beliefs: the property that the agent does not simultaneously believe a proposition and also its negation; that is, the following is a theorem of logic $\mathbb{L}$ :

$$
B \phi \rightarrow \neg B \neg \phi .
$$

We write $\mathbb{L} \vdash \phi$ to denote the fact that formula $\phi$ is a theorem of logic $\mathbb{L}$.

\section{Proposition 1.}

$$
\mathbb{L} \vdash(B \phi \rightarrow \neg B \neg \phi)
$$

In all the proofs, 'PL' stands for 'Propositional Logic' ${ }^{17}$

\footnotetext{
${ }^{17}$ Note that in Step 5 of the following proof we also implicitly use the theorem $P \phi \leftrightarrow P \neg \neg \phi$ which is obtained from the fact that $\phi \leftrightarrow \neg \neg \phi$ is a tautology, so that - by rule $R_{\mathrm{E}}^{P}-$ we get that $P \phi \leftrightarrow P \neg \neg \phi$ is a theorem. The same applies to $C \phi \leftrightarrow C \neg \neg \phi$.
} 
Proof.
1. $B \phi \leftrightarrow(\neg C \neg \phi \wedge P \phi \wedge \neg P \neg \phi)$
(DefB)
2. $(\neg C \neg \phi \wedge P \phi \wedge \neg P \neg \phi) \rightarrow(P \phi \wedge \neg P \neg \phi)$
(tautology)
3. $(P \phi \wedge \neg P \neg \phi) \rightarrow(P \phi \vee \neg P \neg \phi)$
(tautology)
4. $B \phi \rightarrow(P \phi \vee \neg P \neg \phi)$
$(1,2,3$, PL $)$
5. $B \neg \phi \leftrightarrow(\neg C \phi \wedge P \neg \phi \wedge \neg P \phi)$
6. $(\neg C \phi \wedge P \neg \phi \wedge \neg P \phi) \leftrightarrow \neg(C \phi \vee \neg P \neg \phi \vee P \phi)$
7. $\neg B \neg \phi \leftrightarrow(C \phi \vee \neg P \neg \phi \vee P \phi)$
8. $(P \phi \vee \neg P \neg \phi) \rightarrow(C \phi \vee \neg P \neg \phi \vee P \phi)$
(tautology)
9. $B \phi \rightarrow(C \phi \vee \neg P \neg \phi \vee P \phi)$
$(4,8$, PL)
10. $B \phi \rightarrow \neg B \neg \phi$.
$(7,9$, PL)

The next proposition shows that the belief operator is a classical operator (that is, the rule of inference $\left(R_{E}\right)$ applies to it).

Proposition 2. The following is a derived rule of inference of logic $\mathbb{L}$ :

$$
\frac{\phi \leftrightarrow \psi}{B \phi \leftrightarrow B \psi}
$$

Proof.

1. $\phi \leftrightarrow \psi$

(Hypothesis)

2. $P \phi \leftrightarrow P \psi$

$\left(1\right.$, rule $\left.R_{E}^{P}\right)$

3. $\neg \phi \leftrightarrow \neg \psi$

$(1, \mathrm{PL})$

4. $P \neg \phi \leftrightarrow P \neg \psi$

$\left(3\right.$, rule $\left.R_{E}^{P}\right)$

5. $\neg P \neg \phi \leftrightarrow \neg P \neg \psi$

6. $C \neg \phi \leftrightarrow C \neg \psi$

$\left(3\right.$, rule $\left.R_{E}^{C}\right)$

7. $\neg C \neg \phi \leftrightarrow \neg C \neg \psi$

(6, PL)

8. $\quad(\neg C \neg \phi \wedge P \phi \wedge \neg P \neg \phi) \leftrightarrow(\neg C \neg \psi \wedge P \psi \wedge \neg P \neg \psi)$

$(2,5,7, \mathrm{PL})$

9. $B \phi \leftrightarrow(\neg C \neg \phi \wedge P \phi \wedge \neg P \neg \phi)$

10. $B \psi \leftrightarrow(\neg C \neg \psi \wedge P \psi \wedge \neg P \neg \psi)$

(DefB)

11. $B \phi \leftrightarrow B \psi$.

$(8,9,10$, PL) $\square$ 


\section{Extensions of logic $\mathbb{L}$}

In this section we consider the following axioms concerning the interaction of $C$ and $P$, which were discussed in the introduction.

$$
\begin{array}{ll}
\left(C P_{1}\right) & C \phi \rightarrow P \phi \\
\left(C P_{2}\right) & C \phi \rightarrow \neg P \neg \phi \\
\left(C P_{3}\right) & \neg P \phi \rightarrow C \neg P \phi \\
\left(C P_{4}\right) & P \phi \rightarrow C P \phi .
\end{array}
$$

Axiom $\left(C P_{1}\right)$ says that if the agent is certain that $\phi$, then she must consider $\phi$ plausible, while axiom $\left(C P_{2}\right)$ says that if the agent is certain that $\phi$ then she cannot judge the negation of $\phi$ as plausible. Axioms $\left(C P_{3}\right)$ and $\left(C P_{4}\right)$ are introspective properties: the former says that if the agent does not consider $\phi$ plausible, then she is certain that she does not consider $\phi$ plausible, while the latter says that if the agent considers $\phi$ plausible then she is certain that she considers $\phi$ plausible.

The following propositions show that, by extending logic $\mathbb{L}$ to include axioms $\left(C P_{1}\right)-\left(C P_{4}\right)$ we obtain the following properties of belief:

$$
\begin{array}{ll}
B \phi \rightarrow C B \phi & \text { (Belief implies certainty of belief) } \\
\neg B \phi \rightarrow C \neg B \phi & \text { (Absence of belief implies certainty of absence of belief) } \\
B \phi \rightarrow B B \phi & \text { (Positive Introspection of belief) } \\
B B \phi \rightarrow B \phi & \text { (Converse of positive introspection) } \\
\neg B \phi \rightarrow B \neg B \phi & \text { (Negative Introspection of belief) } \\
B \neg B \phi \rightarrow \neg B \phi & \text { (Converse of negative introspection). }
\end{array}
$$

We denote the fact that formula $\phi$ is a theorem of the extension of $\mathbb{L}$ obtained by adding axioms $\phi_{1}, \ldots, \phi_{n}$ as follows:

$$
\mathbb{L}+\left\{\begin{array}{c}
\phi_{1} \\
\cdots \\
\phi_{n}
\end{array}\right\} \vdash \phi
$$

The following proposition shows that Axiom $\left(\mathrm{CP}_{2}\right)$ yields a reduction of $B \phi$ to $(P \phi \wedge \neg P \neg \phi)$. 


\section{Proposition 3.}

$$
\mathbb{L}+\left\{\left(C P_{2}\right) \quad C \phi \rightarrow \neg P \neg \phi\right\} \vdash B \leftrightarrow(P \phi \wedge \neg P \neg \phi)
$$

Proof.

1. $B \phi \leftrightarrow(\neg C \neg \phi \wedge P \phi \wedge \neg P \neg \phi)$

2. $B \phi \rightarrow(P \phi \wedge \neg P \neg \phi)$

3. $C \neg \phi \rightarrow \neg P \phi$ (Axiom $\mathrm{CP}_{2}$ )

5. $P \phi \rightarrow \neg C \neg \phi$

6. $(P \phi \wedge \neg P \neg \phi) \rightarrow(\neg C \neg \phi \wedge P \phi \wedge \neg P \neg \phi)$

7. $(P \phi \wedge \neg P \neg \phi) \rightarrow B \phi$

8. $B \phi \leftrightarrow(P \phi \wedge \neg P \neg \phi)$.

$(2,7, \mathrm{PL})$

The next proposition shows that consistency of certainty (the agent cannot be simultaneously certain of $\phi$ and of $\neg \phi$ ) is provable from the conjunction of Axioms $\left(\mathrm{CP}_{1}\right)$ and $\left(\mathrm{CP}_{2}\right)$.

\section{Proposition 4.}

$$
\mathbb{L}+\left\{\begin{array}{ll}
\left(C P_{1}\right) & C \phi \rightarrow P \phi \\
\left(C P_{2}\right) & C \phi \rightarrow \neg P \neg \phi
\end{array}\right\} \vdash C \phi \rightarrow \neg C \neg \phi
$$

Proof.

$$
\begin{array}{lll}
\text { 1. } & C \neg \phi \rightarrow \neg P \phi & \left(\text { Axiom } \mathrm{CP}_{2}\right) \\
\text { 2. } & P \phi \rightarrow \neg C \neg \phi & (1, \mathrm{PL}) \\
\text { 3. } & C \phi \rightarrow P \phi & \left(\text { Axiom } \mathrm{CP}_{1}\right) \\
\text { 4. } & C \phi \rightarrow \neg C \neg \phi . & (3,2, \mathrm{PL})
\end{array}
$$

The next proposition shows that it is a theorem of the logic $\mathbb{L}+\left\{C P_{1}, C P_{2}\right\}$ that certainty implies belief.

\section{Proposition 5.}

$$
\mathbb{L}+\left\{\begin{array}{ll}
\left(C P_{1}\right) & C \phi \rightarrow P \phi \\
\left(C P_{2}\right) & C \phi \rightarrow \neg P \neg \phi
\end{array}\right\} \vdash C \phi \rightarrow B \phi
$$


The proof is obvious, since the conjunction of the consequents of $\left(C P_{1}\right)$ and $\left(C P_{2}\right)$ is $(P \phi \wedge \neg P \neg \phi)$ which, by Proposition 3 , is equivalent to $B \phi$.

Proposition 6 says that if Axioms $\left(C P_{1}\right),\left(C P_{3}\right)$ and $\left(C P_{4}\right)$ are assumed, then, whenever the agent believes something, she is certain that she believes it and Proposition 7 says that if Axioms $\left(C P_{2}\right),\left(C P_{3}\right)$ and $\left(C P_{4}\right)$ are assumed then, if the agent does not believe $\phi$, then she is certain that she does not believe $\phi$.

\section{Proposition 6.}

$$
\mathbb{L}+\left\{\begin{array}{ll}
\left(C P_{1}\right) & C \phi \rightarrow P \phi \\
\left(C P_{3}\right) & \neg P \phi \rightarrow C \neg P \phi \\
\left(C P_{4}\right) & P \phi \rightarrow C P \phi
\end{array}\right\} \vdash B \rightarrow C B \phi
$$

Proof.

1. $B \phi \leftrightarrow(P \phi \wedge \neg P \neg \phi \wedge \neg C \neg \phi)$

(DefB)

2. $P \phi \rightarrow C P \phi$

(Axiom $\mathrm{CP}_{4}$ )

3. $\neg P \neg \phi \rightarrow C \neg P \neg \phi$ (Axiom $\mathrm{CP}_{3}$ )

4. $B \phi \rightarrow(C P \phi \wedge C \neg P \neg \phi)$

5. $C \neg \phi \rightarrow P \neg \phi$ (Axiom $\mathrm{CP}_{1}$ )

6. $\neg C \neg C \neg \phi \rightarrow \neg C \neg P \neg \phi$

7. $C \neg P \neg \phi \rightarrow C \neg C \neg \phi$

8. $B \phi \rightarrow(C P \phi \wedge C \neg P \neg \phi \wedge C \neg C \neg \phi)$

9. $(C P \phi \wedge C \neg P \neg \phi \wedge C \neg C \neg \phi) \rightarrow C(P \phi \wedge \neg P \neg \phi \wedge \neg C \neg \phi)$

$\left(\mathrm{C}_{\mathrm{C}}:\right.$ Remark 2)

10. $B \phi \rightarrow C(P \phi \wedge \neg P \neg \phi \wedge \neg C \neg \phi)$

11. $C B \phi \leftrightarrow C(P \phi \wedge \neg P \neg \phi \wedge \neg C \neg \phi)$

12. $B \phi \rightarrow C B \phi$.

$(10,11$, PL)

\section{Proposition 7.}

$$
\mathbb{L}+\left\{\begin{array}{ll}
\left(C P_{2}\right) & C \phi \rightarrow \neg P \neg \phi \\
\left(C P_{3}\right) & \neg P \phi \rightarrow C \neg P \phi \\
\left(C P_{4}\right) & P \phi \rightarrow C P \phi
\end{array}\right\} \vdash \neg B \phi \rightarrow C \neg B \phi
$$

Proof.

1. $B \phi \leftrightarrow(P \phi \wedge \neg P \neg \phi)$

(Proposition 3) 

2. $\neg B \phi \leftrightarrow(\neg P \phi \vee P \neg \phi)$
(1, PL)
3. $C \neg B \phi \leftrightarrow C(\neg P \phi \vee P \neg \phi)$
$\left(2, \mathrm{R}_{E}^{C}\right)$
4. $\neg P \phi \rightarrow C \neg P \phi$
(Axiom $\mathrm{CP}_{3}$ )
5. $P \neg \phi \rightarrow C P \neg \phi$
(Axiom $\mathrm{CP}_{4}$ )
6. $\neg B \phi \rightarrow(C \neg P \phi \vee C P \neg \phi)$
$(2,4,5, \mathrm{PL})$
7. $\neg P \phi \rightarrow(\neg P \phi \vee P \neg \phi)$
(tautology)
8. $C \neg P \phi \rightarrow C(\neg P \phi \vee P \neg \phi)$
$\left(7, \mathrm{R}_{K}^{C}\right)$
9. $P \neg \phi \rightarrow(\neg P \phi \vee P \neg \phi)$
(tautology)
10. $C P \neg \phi \rightarrow C(\neg P \phi \vee P \neg \phi)$
$\left(9, R_{K}^{C}\right)$
11. $(C \neg P \phi \vee C P \neg \phi) \rightarrow C(\neg P \phi \vee P \neg \phi)$
$(8,10$, PL)
12. $\neg B \phi \rightarrow C \neg B \phi$.
$(6,11,3$, PL $)$

Next we consider introspection properties of belief, namely,

$$
\begin{array}{ll}
\left(4_{B}\right) & B \phi \rightarrow B B \phi \\
\left(5_{B}\right) & \neg B \phi \rightarrow B \neg B \phi \\
\left(4_{B}^{\mathrm{cnv}}\right) & B B \phi \rightarrow B \phi \\
\left(5_{B}^{\mathrm{cnv}}\right) & B \neg B \phi \rightarrow \neg B \phi .
\end{array}
$$

$\left(4_{B}\right)$ is the property of Positive Introspection of belief, $\left(5_{B}\right)$ is the property of Negative Introspection, $\left(4_{B}^{\text {cnv }}\right)$ is the converse of $\left(4_{B}\right)$ and $\left(5_{B}^{\text {cnv }}\right)$ is the converse of $\left(5_{B}\right)$ (the latter two say that the agent has correct beliefs about what she believes and what she does not believe).

\section{Proposition 8.}

$$
\mathbb{L}+\left\{\begin{array}{ll}
\left(C P_{1}\right) & C \phi \rightarrow P \phi \\
\left(C P_{2}\right) & C \phi \rightarrow \neg P \neg \phi \\
\left(C P_{3}\right) & \neg P \phi \rightarrow C \neg P \phi \\
\left(C P_{4}\right) & P \phi \rightarrow C P \phi
\end{array}\right\} \vdash B \rightarrow B B \phi \text { and } \neg B \phi \rightarrow B \neg B \phi
$$

Proof. Proof of Positive Introspection of belief:
1. $\mathrm{B} \phi \rightarrow \mathrm{CB} \phi$
(Proposition 6)
2. $\mathrm{CB} \phi \rightarrow B B \phi$
(Proposition 5)
3. $B \phi \rightarrow B B \phi$.
$(1,2$, PL) 
Proof of Negative Introspection of belief:
1. $\neg B \phi \rightarrow C \neg B \phi$
(Proposition 7)
2. $C \neg B \phi \rightarrow B \neg B \phi$
(Proposition 5)
3. $\neg B \phi \rightarrow B \neg B \phi$.
$(1,2$, PL )

Remark 4. Note that, in order to obtain positive or negative introspection of belief, we need neither positive nor negative introspection of certainty. Indeed, no axioms at all were imposed on the logic of certainty. The same is true of the properties highlighted in Propositions 5-7.

\section{Proposition 9.}

$$
\mathbb{L}+\left\{\begin{array}{ll}
\left(C_{2}\right) & C \phi \rightarrow \neg P \neg \phi \\
\left(C P_{3}\right) & \neg P \phi \rightarrow C \neg P \phi \\
\left(C P_{4}\right) & P \phi \rightarrow C P \phi
\end{array}\right\} \vdash B B \phi \rightarrow B \phi
$$

Proof.
1. $\neg B \phi \rightarrow C \neg B \phi$
(Proposition 7)
2. $\neg C \neg B \phi \rightarrow B \phi$
(1, PL)
3. $B B \phi \leftrightarrow(\neg C \neg B \phi \wedge P B \phi \wedge \neg P \neg B \phi)$
4. $B B \phi \rightarrow \neg C \neg B \phi$
5. $B B \phi \rightarrow B \phi$.
$(4,2$, PL)

Proposition 10.

$$
\mathbb{L}+\left\{\begin{array}{ll}
\left(C P_{1}\right) & C \phi \rightarrow P \phi \\
\left(C P_{3}\right) & \neg P \phi \rightarrow C \neg P \phi \\
\left(C P_{4}\right) & P \phi \rightarrow C P \phi
\end{array}\right\} \vdash B \neg B \phi \rightarrow \neg B \phi
$$


Proof.
1. $B \neg B \phi \leftrightarrow(\neg C B \phi \wedge P \neg B \phi \wedge \neg P B \phi)$
(DefB)
2. $\neg B \neg B \phi \leftrightarrow(C B \phi \vee \neg P \neg B \phi \vee P B \phi)$
3. $B \phi \rightarrow C B \phi$
(Proposition 6)
4. $C B \phi \rightarrow(C B \phi \vee \neg P \neg B \phi \vee P B \phi)$
(tautology)
5. $B \phi \rightarrow \neg B \neg B \phi$
6. $B \neg B \phi \rightarrow \neg B \phi$.

In Section 7 we show that some of the schemata considered in this section can also be obtained from alternative extensions of logic $\mathbb{L}$. In the next section we turn to the semantics.

\section{Semantics, modal correspondence and minimality}

Definition 4.1. A frame is a triple $\langle\Omega, C, \mathcal{P}\rangle$ where

- $\Omega$ is a non-empty set of states; the subsets of $\Omega$ will be called events or propositions.

- $C \subseteq \Omega \times \Omega$ is a binary relation on $\Omega$, representing "certainty". The interpretation of $\omega C \omega^{\prime}$ is that if the true, or actual, state is $\omega$ then the agent cannot rule out the possibility that the true state is $\omega^{\prime}$. We denote by $C(\omega)=\left\{\omega^{\prime} \in \Omega: \omega C \omega^{\prime}\right\}$ the set of states that the agent cannot rule out at state $\omega$.

- $\mathcal{P}: \Omega \rightarrow 2^{2^{\Omega}}$ (where $2^{\Omega}$ is the set of subsets of $\Omega$ and $2^{2^{\Omega}}$ is the set of subsets of $2^{\Omega}$ ) is a "plausibility" function that associates with every state a collection of events. The interpretation of $E \in \mathcal{P}(\omega)$ is that at state $\omega$ the agent "considers event $E$ plausible". The function $\mathcal{P}$ is known in modal logic as a neighborhood function (see, for example, Pacuit (2017)).

For example, suppose that $\Omega=\left\{\omega_{1}, \omega_{2}, \ldots, \omega_{7}\right\}, C\left(\omega_{1}\right)=\left\{\omega_{4}, \omega_{5}, \omega_{6}, \omega_{7}\right\}$ and $\mathcal{P}\left(\omega_{1}\right)=\left\{\left\{\omega_{4}, \omega_{5}\right\}, \Omega\right\}$. Then, if the true state is $\omega_{1}$, the agent (erroneously) rules out the possibility that the state is $\omega_{1}$ as well as (correctly) the possibility that the true state is either $\omega_{2}$ or $\omega_{3}$; moreover - although she does not rule out the 
possibility that the true state is either $\omega_{6}$ or $\omega_{7}$ - she judges these two states to be implausible. Furthermore, since $\left\{\omega_{4}, \omega_{5}\right\} \in \mathcal{P}\left(\omega_{1}\right)$ and $\left(\Omega \backslash\left\{\omega_{4}, \omega_{5}\right\}\right) \notin \mathcal{P}\left(\omega_{1}\right)$ then we can say that - at state $\omega_{1}$ - the agent believes that the true state is either $\omega_{4}$ or $\omega_{5}$ : this is the semantic counterpart of Definition 2.1 in Section 2 (see the validation rule for $B \phi$ below).

The connection between syntax and semantics is given by the notion of model. Given a frame $\langle\Omega, C, \mathcal{P}\rangle$, a model based on it is obtained by adding a valuation $V:$ At $\rightarrow 2^{\Omega}$ which associates with every atomic proposition $p \in$ At the set of states at which $p$ is true. The truth of an arbitrary formula at a state is then defined inductively as follows $(\omega \models \phi$ denotes that formula $\phi$ is true at state $\omega$; $\|\phi\|$ denotes the truth set of $\phi$, that is, $\|\phi\|=\{\omega \in \Omega: \omega \vDash \phi\})$ :

if $q$ is an atomic proposition, $\omega \vDash q$ if and only if $\omega \in V(q)$

$\omega \vDash \neg \phi$ if and only if $\omega \not \models \phi$

$\omega \vDash \phi \vee \psi$ if and only if either $\omega \vDash \phi$ or $\omega \vDash \psi$ (or both)

$\omega \vDash C \phi$ if and only if $C(\omega) \subseteq\|\phi\|$

$\omega \vDash P \phi$ if and only if $\|\phi\| \in \mathcal{P}(\omega)$

$\omega \models B \phi$ if and only if:

1. $C(\omega) \cap\|\phi\| \neq \varnothing$,

2. $\|\phi\| \in \mathcal{P}(\omega)$, and

3. $(\Omega \backslash\|\phi\|) \notin \mathcal{P}(\omega)$.

It follows that $\omega \vDash \neg C \neg \phi$ if and only if $C(\omega) \cap\|\phi\| \neq \varnothing$ and $\omega \models \neg P \neg \phi$ if and only if $(\Omega \backslash\|\phi\|) \notin \mathcal{P}(\omega)$ (since $\|\neg \phi\|=(\Omega \backslash\|\phi\|)$.

We say that a formula $\phi$ is valid in a model if $\omega \models \phi$ for all $\omega \in \Omega$, that is, if $\phi$ is true at every state in that model. A formula $\phi$ is valid in a frame if it is valid in every model based on that frame. Finally, we say that a property of frames is characterized by (or characterizes) an axiom if (1) the axiom is valid in any frame that satisfies the property and, conversely, (2) if a frame violates the property then there is a model based on that frame such that the axiom is not valid in that model.

The next proposition identifies the semantic properties that characterize the axioms considered in Section 3. 
Proposition 11. Concerning the interaction axioms between certainty and plausibility,

1. Axiom $\left(C P_{1}\right)(C \phi \rightarrow P \phi)$ is characterized by the following property: $\forall \omega \in \Omega, \forall E \in 2^{\Omega}$,

$$
\text { if } C(\omega) \subseteq E \text { then } E \in \mathcal{P}(\omega) \text {. }
$$

2. Axiom $\left(\mathrm{CP}_{2}\right)(\mathrm{C} \phi \rightarrow \neg P \neg \phi)$ is characterized by the following property: $\forall \omega \in \Omega, \forall E \in 2^{\Omega}$,

$$
\text { if } C(\omega) \subseteq E \text { then }(\Omega \backslash E) \notin \mathcal{P}(\omega) \text {. }
$$

3. Axiom $\left(\mathrm{CP}_{3}\right)(\neg P \phi \rightarrow C \neg P \phi)$ is characterized by the following property: $\forall \omega, \omega^{\prime} \in \Omega$,

$$
\text { if } \omega^{\prime} \in C(\omega) \text { then } \mathcal{P}\left(\omega^{\prime}\right) \subseteq \mathcal{P}(\omega) \text {. }
$$

4. Axiom $\left(\mathrm{CP}_{4}\right)(\mathrm{P} \phi \rightarrow \mathrm{CP} \phi)$ is characterized by the following property: $\forall \omega, \omega^{\prime} \in \Omega$,

$$
\text { if } \omega^{\prime} \in \mathcal{C}(\omega) \text { then } \mathcal{P}(\omega) \subseteq \mathcal{P}\left(\omega^{\prime}\right) \text {. }
$$

Proof.

1. Fix an arbitrary model based on a frame that satisfies the property that, $\forall \omega \in \Omega, \forall E \in 2^{\Omega}$, if $C(\omega) \subseteq E$ then $E \in \mathcal{P}(\omega)$. Fix an arbitrary $\omega \in \Omega$ and an arbitrary formula $\phi$ and suppose that $\omega \vDash C \phi$, that is, $C(\omega) \subseteq\|\phi\|$. Then, by the assumed property, $\|\phi\| \in \mathcal{P}(\omega)$, that is, $\omega \vDash P \phi$. Conversely, fix a frame that violates the property, that is, there is an $\omega \in \Omega$ and an $E \in 2^{\Omega}$ such that $C(\omega) \subseteq E$ and $E \notin \mathcal{P}(\omega)$. Let $q$ be an atomic formula and construct a model where $\|q\|=E$. Then $\omega \vDash C q$ and, since $\|q\| \notin \mathcal{P}(\omega)$, $\omega \vDash P q$ (that is, $\omega \vDash \neg P q$ ), so that Axiom $\left(\mathrm{CP}_{1}\right)$ is not valid in this model.

2. Fix an arbitrary model based on a frame that satisfies the property that, $\forall \omega \in \Omega, \forall E \in 2^{\Omega}$, if $C(\omega) \subseteq E$ then $(\Omega \backslash E) \notin \mathcal{P}(\omega)$. Fix an arbitrary $\omega \in \Omega$ and an arbitrary formula $\phi$ and suppose that $\omega \vDash C \phi$, that is, $C(\omega) \subseteq\|\phi\|$. Then, by the assumed property, $(\Omega \backslash\|\phi\|) \notin \mathcal{P}(\omega)$, that is, $\omega \forall P \neg \phi$ or, equivalently, $\omega \vDash \neg P \neg \phi$. Conversely, fix a frame that violates the property, that is, there is an $\omega \in \Omega$ and an $E \in 2^{\Omega}$ such that $C(\omega) \subseteq E$ and $(\Omega \backslash E) \in \mathcal{P}(\omega)$. Let $q$ be an atomic formula and construct a model where $\|q\|=E$. Then $\omega \vDash C q$ and, since $\|\neg q\|=(\Omega \backslash E) \in \mathcal{P}(\omega), \omega \vDash P \neg q$, so that Axiom $\left(\mathrm{CP}_{2}\right)$ is not valid in this model. 
3. Fix an arbitrary model based on a frame that satisfies the property that, $\forall \omega, \omega^{\prime} \in \Omega$, if $\omega^{\prime} \in \mathcal{C}(\omega)$ then $\mathcal{P}\left(\omega^{\prime}\right) \subseteq \mathcal{P}(\omega)$. Fix an arbitrary $\omega \in \Omega$ and an arbitrary formula $\phi$ and suppose that $\omega \vDash \neg P \phi$, that is, $\|\phi\| \notin \mathcal{P}(\omega)$. Fix an arbitrary $\omega^{\prime} \in \mathcal{C}(\omega)$. Then, by the assumed property, $\|\phi\| \notin \mathcal{P}\left(\omega^{\prime}\right)$, so that $\omega^{\prime} \vDash P \phi$, that is, $\omega^{\prime} \vDash \neg P \phi$, and thus, since $\omega^{\prime} \in C(\omega)$ was chosen arbitrarily, $\omega \vDash C \neg P \phi$. Conversely, fix a frame that violates the property, that is, there exist $\omega, \omega^{\prime} \in \Omega$ and $E \in 2^{\Omega}$ such that (a) $\omega^{\prime} \in C(\omega)$, (b) $E \in \mathcal{P}\left(\omega^{\prime}\right)$ and (c) $E \notin \mathcal{P}(\omega)$. Let $q$ be an atomic formula and construct a model where $\|q\|=E$. Then, by (c), $\omega \vDash \neg P q$ while, by (b), $\omega^{\prime} \vDash P q$ so that, since $\omega^{\prime} \in C(\omega), \omega \not \models C \neg P q$ (that is, $\omega \vDash \neg C \neg P q$ ), thus invalidating axiom $\left(\mathrm{CP}_{3}\right)$.

4. Fix an arbitrary model based on a frame that satisfies the property that, $\forall \omega, \omega^{\prime} \in \Omega$, if $\omega^{\prime} \in \mathcal{C}(\omega)$ then $\mathcal{P}(\omega) \subseteq \mathcal{P}\left(\omega^{\prime}\right)$. Fix an arbitrary $\omega \in \Omega$ and an arbitrary formula $\phi$ and suppose that $\omega \vDash P \phi$, that is, $\|\phi\| \in$ $\mathcal{P}(\omega)$. Fix an arbitrary $\omega^{\prime} \in \mathcal{C}(\omega)$. Then, by the assumed property, $\|\phi\| \in$ $\mathcal{P}\left(\omega^{\prime}\right)$, so that $\omega^{\prime} \vDash P \phi$ and thus, since $\omega^{\prime} \in \mathcal{C}(\omega)$ was chosen arbitrarily, $\omega \vDash C P \phi$. Conversely, fix a frame that violates the property, that is, there exist $\omega, \omega^{\prime} \in \Omega$ and $E \in 2^{\Omega}$ such that (a) $\omega^{\prime} \in C(\omega)$, (b) $E \in \mathcal{P}(\omega)$ and (c) $E \notin \mathcal{P}\left(\omega^{\prime}\right)$. Let $q$ be an atomic formula and construct a model where $\|q\|=E$. Then, by (b), $\omega \vDash P q$ while, by (c), $\omega^{\prime} \not \models P q$ (that is, $\omega \vDash \neg P q$ ) so that, since $\omega^{\prime} \in C(\omega), \omega \not \models C P q$, thus invalidating axiom $\left(\mathrm{CP}_{4}\right)$.

A logic is said to be sound with respect to a class of frames if every theorem of the logic is valid in every frame of that class. Since Axiom $\left(\mathrm{K}_{C}\right)$ is valid on all the frames considered here and the rules of inference $(\mathrm{MP}),\left(\mathrm{Nec}_{C}\right)$ and $\left(\mathrm{R}_{\mathrm{E}}^{P}\right)$ are validity preserving, ${ }^{18}$ it follows that logic $\mathbb{L}$ is sound with respect to the class of all frames. The following proposition then follows from Proposition 11.

Proposition 12. Let $\left\{\phi_{1}, \ldots, \phi_{n}\right\}(n \geq 0)$ be any collection of axioms from the set $\left\{C P_{1}, C P_{2}, C P_{3}, C P_{4}\right\}$. Then $\mathbb{L}+\left\{\phi_{1}, \ldots, \phi_{n}\right\}$ is sound with respect to the class of frames that satisfy the collection of properties $\left\{F_{1}, \ldots, F_{n}\right\}$ where, for every $i=1, \ldots, n$, $F_{i}$ is the property that characterizes axiom $\phi_{i}$ (see Proposition 11).

\footnotetext{
${ }^{18}$ The proof that $\left(\mathrm{R}_{\mathrm{E}}^{P}\right)$ is validity preserving is straightforward. For completeness we provide it here. Consider an arbitrary model and suppose that $\phi \leftrightarrow \psi$ is valid, that is, $\|\phi \leftrightarrow \psi\|=\Omega$. Since $\|\phi \leftrightarrow \psi\|=((\Omega \backslash\|\phi\|) \cup\|\psi\|) \cap(\|\phi\| \cup(\Omega \backslash\|\psi\|))$, we have that $((\Omega \backslash\|\phi\|) \cup\|\psi\|)=\Omega$, that is, $\|\phi\| \subseteq\|\psi\|$ and $(\|\phi\| \cup(\Omega \backslash\|\psi\|))=\Omega$, that is, $\|\psi\| \subseteq\|\phi\|$. Thus $\|\phi\|=\|\psi\|$. Fix an arbitrary $\omega \in \Omega$. Then $\omega \vDash P \phi$ if and only if $\|\phi\| \in \mathcal{P}(\omega)$ if and only if $\|\psi\| \in \mathcal{P}(\omega)$ if and only if $\omega \vDash P \psi$, so that $\omega \vDash(P \phi \leftrightarrow P \psi)$. Thus $\|P \phi \leftrightarrow P \psi\|=\Omega$.
} 
For example, the logic $\mathbb{L}+\left\{C P_{3}, C P_{4}\right\}$ is sound with respect to the class of frames where the following property holds: $\forall \omega, \omega^{\prime} \in \Omega$, if $\omega^{\prime} \in C(\omega)$ then $\mathcal{P}\left(\omega^{\prime}\right)=\mathcal{P}(\omega)$.

Definition 4.2. Let $S$ be a non-empty set of axioms and $\phi$ a formula. We say that the logic $\mathbb{L}+S$ is a minimal extension of $\mathbb{L}$ that yields $\phi$ as a theorem if (1) $\mathbb{L}+S \vdash \phi$ and (2) for every proper subset $S^{\prime}$ of $S, \phi$ is not a theorem of $\mathbb{L}+S^{\prime}$.

Remark 5. In virtue of Proposition 12, to show that $\mathbb{L}+\left\{\phi_{1}, \ldots, \phi_{n}\right\}$ is a minimal extension of $\mathbb{L}$ that yields axiom $\phi$ as a theorem, it is sufficient to show that, for every $i=1, \ldots, n, \phi$ is not valid in the class of frames that satisfy the collection of properties $\left\{F_{1}, \ldots, F_{i-1}, F_{i+1}, \ldots, F_{n}\right\}$.

For example, let us show that logic $\mathbb{L}+\left\{C P_{1}, C P_{2}\right\}$ is a minimal extension of $\mathbb{L}$ that yields the theorem $C \phi \rightarrow B \phi$ (see Proposition 5). First of all, by Proposition 12, this logic is sound with respect to the class of frames where properties $\left(\mathrm{F}_{\mathrm{CP}_{1}}\right)$ and $\left(\mathrm{F}_{\mathrm{CP}_{2}}\right)$ are satisfied. In both of the following examples we take $\Omega=\{\alpha, \beta, \gamma\}{ }^{19}$

$\backslash C P_{1}$ Consider the following frame: $C(\alpha)=C(\beta)=C(\gamma)=\{\beta\}, \mathcal{P}(\alpha)=\mathcal{P}(\beta)=$ $\mathcal{P}(\gamma)=\{\{\beta\}, \Omega\}$. This frame satisfies Property $\left(\mathrm{F}_{C P_{2}}\right)$ (thus it validates the logic $\left.\mathbb{L}+\left\{C P_{2}\right\}\right)$, but fails Property $\left(\mathrm{F}_{C P_{1}}\right)$, since $C(\alpha)=\{\beta\} \subseteq\{\beta, \gamma\}$ and yet $\{\beta, \gamma\} \notin \mathcal{P}(\alpha)$. Let $p$ be an atomic formula and consider a model where $\|p\|=\{\beta, \gamma\}$. Then $\alpha \vDash C p$ but $\alpha \not \models B p$ since $\alpha \not \models P p$ (because $\|p\|=\{\beta, \gamma\} \notin \mathcal{P}(\alpha))$.

$\backslash C P_{2}$ Consider the following frame: $C(\alpha)=C(\beta)=C(\gamma)=\{\beta, \gamma\}, \mathcal{P}(\alpha)=\mathcal{P}(\beta)=$ $\mathcal{P}(\gamma)=\{\{\alpha\},\{\beta, \gamma\}, \Omega\}$. This frame satisfies Property $\left(\mathrm{F}_{C P_{1}}\right)$ (thus it validates the logic $\left.\mathbb{L}+\left\{C P_{1}\right\}\right)$, but fails Property $\left(\mathrm{F}_{C P_{2}}\right)$, since $C(\alpha) \subseteq\{\beta, \gamma\}$ and yet $\Omega \backslash\{\beta, \gamma\}=\{\alpha\} \in \mathcal{P}(\alpha)$. Let $p$ be an atomic formula and consider a model where $\|p\|=\{\beta, \gamma\}$. Then $\alpha \vDash C p$ but $\alpha \not \models B p$ since $\alpha \not \models \neg P \neg p$ (because $\|\neg p\|=\Omega \backslash\|p\|=\{\alpha\} \in \mathcal{P}(\alpha)$, so that $\alpha \vDash P \neg p)$.

Since, as the above example shows, it is rather laborious to prove that an extension of logic $\mathbb{L}$ is a minimal extension that yields a particular theorem, the proof of the following proposition is relegated to the Appendix.

Proposition 13. The extensions of logic $\mathbb{L}$ considered in Propositions 5-10 are minimal extensions for the corresponding theorems.

\footnotetext{
${ }^{19}$ Note that, in both frames considered below, $C$ is transitive and Euclidean (these two properties are defined in Remark 6 in the next section) and thus both frames validate both positive and negative introspection of certainty: see Remark 6 .
} 
In the next section we consider the circumstances under which belief satisfies conjunction properties.

\section{Conjunction properties of belief}

In this section we consider the following conjunction properties of belief:

$$
\begin{aligned}
\left(M_{B}\right) & B(\phi \wedge \psi) \rightarrow(B \phi \wedge B \psi) \\
\left(C_{B}^{\text {weak }}\right) & (B \phi \wedge B \psi \wedge \neg C \neg(\phi \wedge \psi)) \rightarrow B(\phi \wedge \psi)
\end{aligned}
$$

$\left(M_{B}\right)$ says that belief in the conjunction of $\phi$ and $\psi$ implies belief in each of $\phi$ and $\psi$, while $\left(C_{B}^{\text {weak }}\right)$ is a weak converse: if the agent believes each of $\phi$ and $\psi$ and does not rule out $(\phi \wedge \psi)$ then she must believe $(\phi \wedge \psi)$.

First we show that the extensions of logic $\mathbb{L}$ considered so far are consistent with the failure of both $\left(M_{B}\right)$ and $\left(C_{B}^{\text {weeak }}\right)$. In order to stress that this is not due to not having restricted the logic of certainty, we will show that this fact remains true even if axioms $T_{C}$ and $5_{C}$ below are added to logic $\mathbb{L}$ (so that certainty can be interpreted as knowledge). First we recall the following possible axioms for certainty:

$\left(D_{C}\right) \quad$ Consistency of certainty: $C \phi \rightarrow \neg C \neg \phi$

$\left(4_{C}\right)$ Positive Introspection of certainty: $C \phi \rightarrow C C \phi$

$\left(5_{C}\right)$ Negative Introspection of certainty: $\neg C \phi \rightarrow C \neg C \phi$

$\left(T_{C}\right)$ Truth of certainty: $C \phi \rightarrow \phi$.

Consistency rules out the possibility that the agent may simultaneously be certain of a proposition and also of its negation. Positive Introspection says that if the agent is certain of $\phi$ then she is certain that she is certain of $\phi$. Negative Introspection says that if the agent is not certain of $\phi$ then she is certain that she is not certain of $\phi$ and Truth says that the agent cannot be certain of something which is not true.

The certainty operator is said to satisfy the S5 logic if it satisfies Axioms $\left(T_{C}\right)$ and $\left(5_{C}\right)$ (in which case $\left(D_{C}\right)$ and $\left(4_{C}\right)$ can be derived as theorems) and is said to satisfy the KD45 logic if it satisfies Axioms $\left(D_{C}\right),\left(4_{C}\right)$ and $\left(5_{C}\right)$.

Remark 6. It is well-known that the semantic properties corresponding to the above axioms are as follows: 
1. $\left(D_{C}\right)$ is characterized by seriality of the relation $C: \forall \omega \in \Omega$,

$$
C(\omega) \neq \varnothing .
$$

2. $\left(4_{C}\right)$ is characterized by transitivity of the relation $C: \forall \omega, \omega^{\prime} \in \Omega$,

$$
\text { if } \omega^{\prime} \in \mathcal{C}(\omega) \text { then } \mathcal{C}\left(\omega^{\prime}\right) \subseteq C(\omega) \text {. }
$$

3. $\left(5_{C}\right)$ is characterized by Euclideanness of the relation $C: \forall \omega, \omega^{\prime} \in \Omega$,

$$
\text { if } \omega^{\prime} \in C(\omega) \text { then } C(\omega) \subseteq C\left(\omega^{\prime}\right) \text {. }
$$

4. $\left(T_{C}\right)$ is characterized by reflexivity of the relation $C: \forall \omega \in \Omega$,

$$
\omega \in C(\omega) .
$$

As Example 1 below shows, none of the extensions of logic $\mathbb{L}$ considered in Section 3 yield $\left(\mathrm{M}_{B}\right)$ as a theorem, even if the logic is further extended by requiring the certainty operator to satisfy the S5 logic. The frame considered in the example validates the logic

$$
\mathbb{L}+ \begin{cases}\left(T_{C}\right) & C \phi \rightarrow \phi \\ \left(5_{C}\right) & \neg C \phi \rightarrow C \neg C \phi \\ \left(C P_{1}\right) & C \phi \rightarrow P \phi \\ \left(C P_{2}\right) & C \phi \rightarrow \neg P \neg \phi \\ \left(C P_{3}\right) & \neg P \phi \rightarrow C \neg P \phi \\ \left(C P_{4}\right) & P \phi \rightarrow C P \phi\end{cases}
$$

Example 1 (violation of $\mathrm{M}_{B}$ ). Consider the following frame: $\Omega=\{\alpha, \beta, \gamma\}$, $\mathcal{C}(\alpha)=\mathcal{C}(\beta)=\mathcal{C}(\gamma)=\Omega, \mathcal{P}(\alpha)=\mathcal{P}(\beta)=\mathcal{P}(\gamma)=\{\{\beta\}, \Omega\} .{ }^{20}$ Let $p$ and $q$ be atomic propositions and construct a model where $\|p\|=\{\alpha, \beta\}$ and $\|q\|=\{\beta, \gamma\}$. Then $\|p \wedge q\|=\{\beta\}$ and $\beta \models B(p \wedge q)($ since $C(\beta) \cap\|p \wedge q\|=\{\beta\} \neq \varnothing,\|p \wedge q\| \in \mathcal{P}(\beta)$ and $(\Omega \backslash\|p \wedge q\|)=\{\alpha, \gamma\} \notin \mathcal{P}(\beta))$; however $\beta \not \forall B p$ because $\beta \not p P p$ (since $\|p\|=\{\alpha, \beta\} \notin \mathcal{P}(\beta)$; similarly, $\beta \not \models B q)$.

\footnotetext{
${ }^{20} \mathrm{C}$ is reflexive and Euclidean (and thus serial and transitive) and Properties $\mathrm{F}_{C P_{1}}, \mathrm{~F}_{C P_{2}}, \mathrm{~F}_{C P_{3}}$, and $\mathrm{F}_{C P_{4}}$ are all satisfied. Note that, in this example, Property $\mathrm{F}_{C P_{1}}$ only requires that $\Omega \in \mathcal{P}(\omega)$, $\forall \omega \in \Omega$, and Property $\mathrm{F}_{\mathrm{CP}_{2}}$ only requires that $\varnothing \notin \mathcal{P}(\omega), \forall \omega \in \Omega$.
} 
The reason why the frame considered in Example 1 fails to validate $\left(\mathrm{M}_{B}\right)$ is that, although event $\{\beta\}$ is considered plausible, no superset of it (with the exception of $\Omega$ ) is considered plausible, that is, the frame violates the following property ('MON' stands for 'Monotonicity'):

$$
\forall \omega \in \Omega, \forall E, F \in 2^{\Omega} \text {, if } E \subseteq F \text { and } E \in \mathcal{P}(\omega) \text { then } F \in \mathcal{P}(\omega) .
$$

Consider the following axioms:

$$
\begin{aligned}
& P(\phi \wedge \psi) \rightarrow(P \phi \wedge P \psi) \\
& (P \phi \vee P \psi) \rightarrow P(\phi \vee \psi) .
\end{aligned}
$$

Axiom $\left(P_{1}\right)$ requires that if the conjunction of $\phi$ and $\psi$ is considered plausible then each of $\phi$ and $\psi$ should be considered plausible, while Axiom $\left(P_{2}\right)$ says that if either $\phi$ or $\psi$ is considered plausible then their disjunction is also considered plausible.

Remark 7. Axioms $\left(P_{1}\right)$ and $\left(P_{2}\right)$ are valid on every frame that satisfies Property $(\mathrm{MON})^{21}$

The next proposition shows that $\left(\mathrm{M}_{B}\right)$ is a theorem of $\mathbb{L}+\left\{P_{1}, P_{2}\right\}$.

\section{Proposition 14.}

$$
\mathbb{L}+\left\{\begin{array}{ll}
\left(P_{1}\right) & P(\phi \wedge \psi) \rightarrow(P \phi \wedge P \psi) \\
\left(P_{2}\right) & (P \phi \vee P \psi) \rightarrow P(\phi \vee \psi)
\end{array}\right\} \vdash B(\phi \wedge \phi) \rightarrow(B \phi \wedge B \psi)
$$

Proof.

1. $B(\phi \wedge \psi) \leftrightarrow(\neg C \neg(\phi \wedge \psi) \wedge P(\phi \wedge \psi) \wedge \neg P \neg(\phi \wedge \psi))$

2. $\neg C \neg(\phi \wedge \psi) \rightarrow(\neg C \neg \phi \wedge \neg C \neg \psi)$

3. $P(\phi \wedge \psi) \rightarrow(P \phi \wedge P \psi)$

${ }^{21}$ Proof of validity of $\left(P_{1}\right)$ : fix an arbitrary frame that satisfies $(\mathrm{MON})$, an arbitrary model based on it, arbitrary formulas $\phi$ and $\psi$ and an arbitrary state $\omega$. Suppose that $\omega \vDash P(\phi \wedge \psi)$, that is, $\|\phi \wedge \psi\| \in \mathcal{P}(\omega)$. Since $\|\phi \wedge \psi\|=\|\phi\| \cap\|\psi\| \subseteq\|\phi\|$, by (MON) $\|\phi\| \in \mathcal{P}(\omega)$ and thus $\omega \models P \phi$. Similarly, since $\|\phi\| \cap\|\psi\| \subseteq\|\psi\|$, by (MON) $\|\psi\| \in \mathcal{P}(\omega)$ and thus $\omega \models P \psi$. Hence $\omega \vDash(P \phi \wedge P \psi)$.

Proof of validity of $\left(P_{2}\right)$ : fix an arbitrary frame that satisfies (MON), an arbitrary model based on it, arbitrary formulas $\phi$ and $\psi$ and an arbitrary state $\omega$. Suppose that $\omega \models(P \phi \vee P \psi)$, that is, either $\|\phi\| \in \mathcal{P}(\omega)$ or $\|\psi\| \in \mathcal{P}(\omega)$. If $\|\phi\| \in \mathcal{P}(\omega)$, since $\|\phi\| \subseteq\|\phi\| \cup\|\psi\|=\|\phi \vee \psi\|$, by (MON) $\|\phi \vee \psi\| \in \mathcal{P}(\omega)$ and thus $\omega \vDash P(\phi \vee \psi)$. The argument for the case where $\|\psi\| \in \mathcal{P}(\omega)$ is similar. Thus $\omega \vDash P(\phi \vee \psi)$. 
4. $\quad(\neg \phi \vee \neg \psi) \leftrightarrow \neg(\phi \wedge \psi)$

(tautology)

5. $\quad P(\neg \phi \vee \neg \psi) \leftrightarrow P \neg(\phi \wedge \psi)$

$\left(4, \mathrm{R}_{\mathrm{E}}^{P}\right)$

6. $(P \neg \phi \vee P \neg \psi) \rightarrow P(\neg \phi \vee \neg \psi)$

(Axiom $P_{2}$ )

7. $(P \neg \phi \vee P \neg \psi) \rightarrow P \neg(\phi \wedge \psi)$

$(6,5, \mathrm{PL})$

8. $\neg P \neg(\phi \wedge \psi) \rightarrow(\neg P \neg \phi \wedge \neg P \neg \psi)$

9. $(\neg C \neg(\phi \wedge \psi) \wedge P(\phi \wedge \psi) \wedge \neg P \neg(\phi \wedge \psi))$

$\rightarrow(\neg C \neg \phi \wedge P \phi \wedge \neg P \neg \phi)$

10. $(\neg C \neg(\phi \wedge \psi) \wedge P(\phi \wedge \psi) \wedge \neg P \neg(\phi \wedge \psi))$

$\rightarrow(\neg C \neg \psi \wedge P \psi \wedge \neg P \neg \psi)$

11. $B \phi \leftrightarrow(\neg C \neg \phi \wedge P \phi \wedge \neg P \neg \phi)$

12. $B \psi \leftrightarrow(\neg C \neg \psi \wedge P \psi \wedge \neg P \neg \psi)$

13. $B(\phi \wedge \psi) \rightarrow(B \phi \wedge B \psi)$

$(1,9,10,11,12$, PL).

The following example shows that $\left(C_{B}^{\text {weak }}\right)$ is not a theorem of any extension of $\mathbb{L}+\left\{\left(P_{1}\right)+\left(P_{2}\right)\right\}$ obtained by adding any subset of the set of axioms $\left\{D_{C}, 4_{C}, 5_{C}, T_{C}\right.$, $\left.C P_{i}(i=1, \ldots, 4)\right\}$ : indeed, the frame considered in Example 2 validates the logic

$$
\mathbb{L}+ \begin{cases}\left(T_{C}\right) & C \phi \rightarrow \phi \\ \left(5_{C}\right) & \neg C \phi \rightarrow C \neg C \phi \\ \left(C P_{1}\right) & C \phi \rightarrow P \phi \\ \left(C P_{2}\right) & C \phi \rightarrow \neg P \neg \phi \\ \left(C P_{3}\right) & \neg P \phi \rightarrow C \neg P \phi \\ \left(C P_{4}\right) & P \phi \rightarrow C P \phi \\ \left(P_{1}\right) & P(\phi \wedge \psi) \rightarrow(P \phi \wedge P \psi) \\ \left(P_{2}\right) & (P \phi \vee P \psi) \rightarrow P(\phi \vee \psi)\end{cases}
$$

Example 2 (violation of $C_{B}^{\text {weak }}$ ). Consider the following frame: $\Omega=\{\alpha, \beta, \gamma\}$, $\mathcal{C}(\alpha)=\mathcal{C}(\beta)=\mathcal{C}(\gamma)=\Omega, \mathcal{P}(\alpha)=\mathcal{P}(\beta)=\mathcal{P}(\gamma)=\{\{\alpha, \beta\},\{\beta, \gamma\}, \Omega\}{ }^{22}$ Let $p$ and $q$ be atomic propositions and construct a model where $\|p\|=\{\alpha, \beta\}$ and $\|q\|=\{\beta, \gamma\}$. Then $\beta \vDash B p \wedge B q \wedge \neg C \neg(p \wedge q)$ (since $(1) C(\beta) \cap\|p\|=\{\alpha, \beta\} \neq \varnothing,\|p\| \in \mathcal{P}(\beta)$, $(\Omega \backslash\|p\|)=\{\gamma\} \notin \mathcal{P}(\beta)),(2) \mathcal{C}(\beta) \cap\|q\|=\{\beta, \gamma\} \neq \varnothing,\|q\| \in \mathcal{P}(\beta),(\Omega \backslash\|q\|)=\{\alpha\} \notin \mathcal{P}(\beta)$ and (3) $C(\beta) \cap\|p \wedge q\|=\{\beta\} \neq \varnothing)$; however $\beta \not \models P(p \wedge q)$ since $\|p \wedge q\|=\{\beta\} \notin \mathcal{P}(\beta)$.

\footnotetext{
${ }^{22} \mathrm{C}$ is reflexive and Euclidean, Properties $\mathrm{F}_{\mathrm{CP}_{1}}, \mathrm{~F}_{\mathrm{CP}_{2}}, \mathrm{~F}_{\mathrm{CP}_{3}}$ and $\mathrm{F}_{\mathrm{CP}_{4}}$ are all satisfied and the frame satisfies Property (MON) (see Remark 7).
} 

axioms:

In order to obtain $\left(C_{B}^{\text {weak }}\right)$ as a theorem we need to add the following two

$$
\begin{gathered}
\neg P \phi \rightarrow P \neg \phi \\
(\neg P \phi \wedge \neg P \psi) \rightarrow \neg P(\phi \vee \psi)
\end{gathered}
$$

Axiom $\left(P_{3}\right)$ says that if $\phi$ is not plausible then its negation must be plausible and Axiom $\left(P_{4}\right)$ says that if neither $\phi$ nor $\psi$ are plausible then their disjunction is not plausible either.

The proof of the following proposition is straightforward and is omitted.

\section{Proposition 15.}

1. Axiom $\left(P_{3}\right)$ is characterized by the following property: $\forall \omega \in \Omega, \forall E \in 2^{\Omega}$,

$$
\text { if } E \notin \mathcal{P}(\omega) \text { then }(\Omega \backslash E) \in \mathcal{P}(\omega) \text {. }
$$

2. Axiom $\left(P_{4}\right)$ is characterized by the following property: $\forall \omega \in \Omega, \forall E, F \in 2^{\Omega}$,

$$
\text { if } E \notin \mathcal{P}(\omega) \text { and } F \notin \mathcal{P}(\omega) \text { then }(E \cup F) \notin \mathcal{P}(\omega) \text {. }
$$

We can now show that, by adding Axioms $\left(\mathrm{P}_{3}\right)$ and $\left(\mathrm{P}_{4}\right)$ to logic $\mathbb{L}$, one obtains $\left(\mathrm{C}_{B}^{\text {weak }}\right)$ as a theorem. First we need the following proposition.

\section{Proposition 16.}

$\mathbb{L}+\left\{\left(P_{4}\right) \quad(\neg P \phi \wedge \neg P \psi) \rightarrow \neg P(\phi \vee \psi)\right\} \vdash(\neg P \neg \phi \wedge \neg P \neg \psi) \rightarrow \neg P \neg(\phi \wedge \psi)$

Proof.
1. $(\neg P \neg \phi \wedge \neg P \neg \psi) \rightarrow \neg P(\neg \phi \vee \neg \psi) \quad\left(\right.$ Axiom $\left.P_{4}\right)$
2. $(\neg \phi \vee \neg \psi) \leftrightarrow \neg(\phi \wedge \psi)$ (tautology)
3. $P(\neg \phi \vee \neg \psi) \leftrightarrow P \neg(\phi \wedge \psi)$
4. $\neg P(\neg \phi \vee \neg \psi) \leftrightarrow \neg P \neg(\phi \wedge \psi)$
5. $(\neg P \neg \phi \wedge \neg P \neg \psi) \rightarrow \neg P \neg(\phi \wedge \psi)$.
(1, 4, PL)

\section{Proposition 17.}

$\mathbb{L}+\left\{\begin{array}{ll}\left(P_{3}\right) & \neg P \phi \rightarrow P \neg \phi \\ \left(P_{4}\right) & (\neg P \phi \wedge \neg P \psi) \rightarrow \neg P(\phi \vee \psi)\end{array}\right\} \vdash B \wedge \wedge B \psi \wedge \neg C \neg(\phi \wedge \psi) \rightarrow B(\phi \wedge \psi)$ 
Proof. ${ }^{23}$

1. $B \phi \leftrightarrow(\neg C \neg \phi \wedge P \phi \wedge \neg P \neg \phi)$

(DefB)

2. $B \psi \leftrightarrow(\neg C \neg \psi \wedge P \psi \wedge \neg P \neg \psi)$

3. $(B \phi \wedge B \psi) \rightarrow(\neg P \neg \phi \wedge \neg P \neg \psi)$

4. $(\neg P \neg \phi \wedge \neg P \neg \psi) \rightarrow \neg P \neg(\phi \wedge \psi)$

(Proposition 16)

5. $(B \phi \wedge B \psi) \rightarrow \neg P \neg(\phi \wedge \psi)$

6. $\neg P \neg(\phi \wedge \psi) \rightarrow P(\phi \wedge \psi)$ (Axiom $P_{3}$ )

7. $(B \phi \wedge B \psi) \rightarrow(\neg P \neg(\phi \wedge \psi) \wedge P(\phi \wedge \psi))$

8. $(B \phi \wedge B \psi \wedge \neg C \neg(\phi \wedge \psi)) \rightarrow$ $\rightarrow(\neg C \neg(\phi \wedge \psi) \wedge P(\phi \wedge \psi) \wedge \neg P \neg(\phi \wedge \psi))$

9. $B(\phi \wedge \psi) \leftrightarrow(\neg C \neg(\phi \wedge \psi) \wedge P(\phi \wedge \psi) \wedge \neg P \neg(\phi \wedge \psi))$

10. $(B \phi \wedge B \psi \wedge \neg C \neg(\phi \wedge \psi)) \rightarrow B(\phi \wedge \psi)$.

$(11,12$, PL $)$

\section{Special cases and related literature}

In this section we first consider several possible interpretations of plausibility, relating our framework to some of the literature, and then discuss other relevant literature.

\subsection{Plausibility as positive probability}

A commonly used semantic structure in game theory (see, for example, Aumann (1976; 1999), Battigalli and Bonanno (1999)) consists of:

1. An equivalence relation $\approx$ on a non-empty, finite ${ }^{24}$ set of states $\Omega$, representing the agent's possible states of knowledge; the interpretation of

${ }^{23}$ Step 6 in the following proof is a shortcut; the full proof of that step is as follows:

$$
\begin{array}{llc}
\text { 1. } & \neg P \neg(\phi \wedge \psi) \rightarrow P \neg \neg(\phi \wedge \psi) & \text { (Axiom } \left.P_{3}\right) \\
\text { 2. } & \neg \neg(\phi \wedge \psi) \leftrightarrow(\phi \wedge \psi) & \text { (tautology) } \\
\text { 3. } & P \neg \neg(\phi \wedge \psi) \leftrightarrow P(\phi \wedge \psi) & \left(2, \text { Rule } R_{E}^{P}\right) \\
\text { 4. } & \neg P \neg(\phi \wedge \psi) \rightarrow P(\phi \wedge \psi) . & (1,3, \text { PL) }
\end{array}
$$

${ }^{24}$ For simplicity we restrict attention to the case where the set of states is finite. 
$\omega \approx \omega^{\prime}$ is that at state $\omega$ the agent considers $\omega^{\prime}$ possible. Letting $[\omega]$ be the equivalence class that contains state $\omega$ (that is, $[\omega]=\left\{\omega^{\prime} \in \Omega: \omega \approx \omega^{\prime}\right\}$ ), we have that the collection $\{[\omega]: \omega \in \Omega\}$ is a partition of $\Omega$.

2. For every state $\omega \in \Omega$, a probability distribution $\mu_{\omega}$ on $\Omega$ such that:

(a) if $\omega \approx \omega^{\prime}$ then $\mu_{\omega}=\mu_{\omega^{\prime}}$ and

(b) if $\mu_{\omega}\left(\omega^{\prime}\right)>0$ then $\omega^{\prime} \approx \omega$.

The probability distribution $\mu_{\omega}$ represents the probabilistic assessment of the agent when her state of knowledge is given by the cell [ $\omega$ ] of her partition. Condition 2a ensures that that the probabilistic assessment is the same at any two states that belong to the same cell of the partition and Condition $2 \mathrm{~b}$ requires the support of $\mu_{\omega}$ to be a subset of the cell $[\omega]{ }^{25}$

Within this framework one can define an event $E \in 2^{\Omega}$ to be plausible at state $\omega$ if it has positive probability, that is, one can define

$$
\mathcal{P}(\omega)=\left\{E \in 2^{\Omega}: \mu_{\omega}(E)>0\right\}
$$

where $\mu_{\omega}(E)=\sum_{x \in E} \mu_{\omega}(x)$. Then the Monotonicity property (MON) is satisfied and so are the properties that characterize Axioms $\left(P_{3}\right)$ and $\left(P_{4}\right)$ (see Proposition 15). With this definition of plausibility, in any model based on such a framework, for every formula $\phi$ and every state $\omega$,

$$
\omega \vDash(P \phi \wedge \neg P \neg \phi) \quad \text { if and only if } \quad \mu_{\omega}(\|\phi\|)=1
$$

that is, if $\sum_{x \in\|\phi\|} \mu_{\omega}(x)=1 .{ }^{26}$ Note that, by Condition 2a above, $\mu_{\omega}(\|\phi\|)=1$ implies that $\|\phi\| \cap[\omega] \neq \varnothing$ and thus, if one identifies the certainty relation $C$ with the

\footnotetext{
${ }^{25}$ Equivalently, one can postulate a probability distribution $\mu: \Omega \rightarrow[0,1]$ such that, $\forall \omega \in \Omega$, $\operatorname{Supp}(\mu) \cap[\omega] \neq \varnothing$, where $\operatorname{Supp}(\mu)=\{\omega \in \Omega: \mu(\omega)>0\}$. Then, for every state $\omega$, one defines $\mu_{\omega}$ to be the probability distribution obtained by conditioning $\mu$ on $[\omega]$, that is,

$$
\mu_{\omega}\left(\omega^{\prime}\right)= \begin{cases}\frac{\mu\left(\omega^{\prime}\right)}{\sum_{x \in[\omega]} \mu(x)} & \text { if } \omega \approx \omega^{\prime} \\ 0 & \text { otherwise. }\end{cases}
$$

${ }^{26}$ If $\omega \vDash P \phi$ then $\|\phi\| \in \mathcal{P}(\omega)$, that is, $\mu_{\omega}(\|\phi\|)>0$ and if $\omega \vDash \neg P \neg \phi$ then $\neg\|\phi\| \notin \mathcal{P}(\omega)$, that is, $\mu_{\omega}(\neg\|\phi\|)=0$ and thus $\mu_{\omega}(\|\phi\|)=1$.
} 
equivalence relation $\approx, \omega \models \neg C \neg \phi$. Hence, by the validation rule for belief, in these structures, believing a proposition at a state coincides with assigning probability 1 to it (at that state). ${ }^{27}$ Identifying the certainty relation $C$ with the equivalence relation $\approx$ and defining plausibility as positive probability, this class of frames validates all the axioms considered in Section 3, as well as Axioms $\left(T_{C}\right),\left(5_{C}\right)$, $\left(P_{1}\right),\left(P_{2}\right),\left(P_{3}\right)$ and $\left(P_{4}\right)$. Hence the logic validated by this class of frameworks is the following extension of $\mathbb{L}$ :

$$
\mathbb{L}+\left\{T_{C}, 5_{C}, C P_{1}, C P_{2}, C P_{3}, C P_{4}, P_{1}, P_{2}, P_{3}, P_{4}\right\} .
$$

All the schemata considered in Propositions 5-17 are theorems of this logic and are thus valid in this class of frames.

\subsection{Plausibility based on a preference order}

A common approach in computer science and philosophy is to postulate a preference order $\gtrsim$ on the set of states, capturing the perceived relative likelihood of states: $\omega \gtrsim \omega^{\prime}$ is interpreted as "state $\omega$ is at least as likely as state $\omega^{\prime}$." Various authors (see, for example, Boutilier (1992), Goldszmidt and Pearl (1992), Spohn (1988)) have then interpreted "the agent believes $\phi$ " as " $\phi$ is true in the most preferred states among those that the agent considers possible". To obtain this interpretation of belief within our approach, let $B e s t_{\gtrsim} C(\omega)$ be the set of most preferred states among the ones that are possible according to $C$ at state $\omega$ :

$$
\text { Best }_{\gtrsim} C(\omega)=\left\{\omega^{\prime} \in C(\omega): \omega^{\prime} \gtrsim x, \forall x \in C(\omega)\right\} .
$$

Define $\mathcal{P}$ as follows: $\forall \omega \in \Omega, \forall E \in 2^{\Omega}$,

$$
E \in \mathcal{P}(\omega) \text { if and only if } E \cap \operatorname{Best}_{\gtrsim} C(\omega) \neq \varnothing
$$

that is, $E$ is judged to be plausible at state $\omega$ if it contains at least one of the most preferred states within the set $C(\omega)$. Then this class of frames validates Axioms

\footnotetext{
${ }^{27}$ Thus, in this case, our derived notion of belief coincides with Lenzen's notion of "strong belief" (Lenzen (2004)). Dodd (2017) argues that (in a probabilistic setting) this ought to be the correct notion of belief:

I argue that believing that $p$ implies having a credence of 1 in $p$. This is true because the belief that $p$ involves representing $p$ as being the case, representing $p$ as being the case involves not allowing for the possibility of not- $p$, while having a credence that's greater than 0 in not- $p$ involves regarding not- $p$ as a possibility.
} 
$\left(C P_{1}\right),\left(C P_{2}\right),\left(P_{1}\right),\left(P_{2}\right),\left(P_{3}\right)$ and $\left(P_{4}\right)$. Thus the corresponding logic will be

$$
\mathbb{L}+\left\{C P_{1}, C P_{2}, P_{1}, P_{2}, P_{3}, P_{4}\right\}+S
$$

where $S$ is the (possibly empty) collection of axioms from the set $\left\{D_{C}, 4_{C}, 5_{C}, T_{C}\right\}$ that are postulated for certainty. Since consistency of certainty (Axiom $D_{C}$ ) is a theorem of this logic (Proposition 4) one obtains the interpretation of belief as "true at the most preferred among the possible states", that is, in every model based on such a framework, $\forall \omega \in \Omega$ and for every formula $\phi$,

$$
\omega \vDash B \phi \text { if and only if } \operatorname{Best}_{\gtrsim} C(\omega) \subseteq\|\phi\| .
$$

\subsection{Plausibility as truth at $100 x \%$ of the accessible states}

Another interpretation of " $\phi$ is plausible" is in terms of the percentage of the accessible states at which $\phi$ is true. Let $x$ be a positive number between 0 and 1. Then " $\phi$ is plausible" is interpreted as " $\phi$ is true at, at least, $100 x \%$ of the accessible states", that is, assuming that $\Omega$ is finite and letting \#F denote the number of elements in event $F \subseteq \Omega$, for every $\omega \in \Omega$ :

$$
\mathcal{P}(\omega)=\left\{E \in 2^{\Omega}: \frac{\#(E \cap C(\omega))}{\# C(\omega)} \geq x\right\} .
$$

Under this interpretation, $\phi$ is believed at state $\omega$ if and only if (1) $\phi$ is true at, at least, $100 x \%$ of the states that are accessible from $\omega$ and (2) $\neg \phi$ is true at less than $100 x \%$ of the states that are accessible from $\omega$, that is, these frames validate the formula $(B \phi \leftrightarrow(P \phi \wedge \neg P \neg \phi)) .{ }^{28}$ Thus belief satisfies one of the conjunction properties, namely $\left(M_{B}\right),{ }^{29}$ but - if $x<1$ - not the other, namely $\left(C_{B}^{\text {weak }}\right) \cdot{ }^{30}$

We can distinguish three cases.

\footnotetext{
${ }^{28} \omega \vDash B \phi$ if and only if (1) $\omega \vDash P \phi$, that is, $\frac{\#(\|\phi\| \cap C(\omega))}{\# C(\omega)} \geq x$, which implies (since $\left.x>0\right)$ that $\|\phi\| \cap C(\omega) \neq \varnothing$, that is, $\omega \models \neg C \neg \phi$, and (2) $\omega \vDash \neg P \neg \phi$, that is, $\frac{\#(\|\neg \phi\| \cap C(\omega))}{\# C(\omega)}<x$.

${ }^{29}$ If $\left.\phi \wedge \psi\right)$ is true at, at least, $100 x \%$ of the states that are accessible from $\omega$ then $\phi$ is true at, at least, $100 x \%$ of the states that are accessible from $\omega$ and so is $\psi$, so that $(P(\phi \wedge \psi) \rightarrow(P \phi \wedge P \psi))$ is valid. If, furthermore, $\neg(\phi \wedge \psi)$ or, equivalently, $(\neg \phi \vee \neg \psi)$ is true at less than $100 x \%$ of the states that are accessible from $\omega$, then $\neg \phi$ is true at less than $100 x \%$ of the states that are accessible from $\omega$ and so is $\neg \psi$, so that $(\neg P \neg(\phi \wedge \psi) \rightarrow(\neg P \neg \phi \wedge \neg P \neg \psi))$ is valid.

${ }^{30}$ If $x<1$ it is possible that (1) $\phi$ is true at, at least, $100 x \%$ of the accessible states and (2) $\psi$ is true at, at least, $100 x \%$ of the accessible states but $\phi \wedge \psi$ is true at some, but less than $100 x \%$, of the accessible states.
} 
1. $x \in\left(0, \frac{1}{2}\right]$. In this case we have that at state $\omega$ the agent believes $\phi$ if and only if $\phi$ is true at more than $(100-x) \%$ of the accessible states, that is,

$$
\omega \models B \phi \text { if and only if } \frac{\#(\|\phi\| \cap C(\omega))}{\# C(\omega)}>1-x .{ }^{31}
$$

For example, if $x=0.2$ then $\phi$ is believed if and only if it is true at more than $80 \%$ of accessible states, ${ }^{32}$ while if $x=0.5$ then $\phi$ is believed if and only if it is true at the majority of accessible states.

The logic validated by this class of frames is

$$
\mathbb{L}+\left\{C P_{1}, C P_{2}, P_{1}, P_{2}, P_{3}\right\}+S
$$

where $S$ is the (possibly empty) collection of axioms from the set $\left\{D_{C}, 4_{C}, 5_{C}\right.$, $\left.T_{C}\right\}$ that are postulated for certainty. Note that Axiom $\left(\mathrm{P}_{4}\right)$ is not valid in this frames. Case 1 (in particular, when $x$ is close to 0 ) is an interesting case since it allows one to capture phenomena such as the Lottery Paradox (Kyburg $(1961 ; 1983)$ ) discussed in the Introduction. ${ }^{33}$

2. $x \in\left(\frac{1}{2}, 1\right)$. In this case we have that the agent believes $\phi$ if and only if the agent considers $\phi$ plausible, ${ }^{34}$ that is, we have the reduction of belief to plausibility: $\omega \vDash B \phi$ if and only $\omega \vDash P \phi$. As in this previous case, the logic validated by this class of frames is

$$
\mathbb{L}+\left\{C P_{1}, C P_{2}, P_{1}, P_{2}, P_{3}\right\}+S
$$

\footnotetext{
${ }^{31}$ First of all, note that when $x \in\left(0, \frac{1}{2}\right],(1-x) \geq x$. Define $A_{\phi}=\|\phi\| \cap C(\omega)$ and $B_{\neg \phi}=\|\neg \phi\| \cap C(\omega)$. Then $\left\{A_{\phi}, B_{\neg \phi}\right\}$ is a partition of $C(\omega)$ so that $\frac{\# A_{\phi}}{\# C(\omega)}+\frac{\# B_{\neg \phi}}{\# C(\omega)}=1$. Let $\phi$ be such that $\frac{\# A_{\phi}}{\# C(\omega)}>(1-x)$. Then $\|\phi\| \cap C(\omega) \neq \varnothing, \quad \frac{\# A_{\phi}}{\# C(\omega)}>x$ (since $1-x \geq x$ ), implying that $\|\phi\| \in \mathcal{P}(\omega)$, and $\frac{\# B_{\neg \phi}}{\# \mathcal{C}(\omega)}<x$, implying that $\|\neg \phi\| \notin \mathcal{P}(\omega)$; hence $\omega \vDash B \phi$. Conversely suppose that $\omega \vDash B \phi$. Then $\omega \models \neg P \neg \phi$, that is, $\frac{\# B_{\neg \phi}}{\# C(\omega)}<x$ and thus, since $\frac{\# A_{\phi}}{\# C(\omega)}=1-\frac{\# B_{\neg \phi}}{\# C(\omega)}, \frac{\# A_{\phi}}{\# C(\omega)}>1-x$.

${ }^{32}$ If $x=0.2$ and, say, $\phi$ is true at $51 \%$ of the states that are accessible from $\omega$, then $\omega \vDash P \phi$ but $\omega \not \models B \phi$ because it is also the case that $\omega \vDash P \neg \phi$ (since $\neg \phi$ is true at $49 \%$ of the states that are accessible from $\omega$ and $0.49>0.2$ ).

${ }^{33}$ For example, suppose that there are three states, $\omega_{1}, \omega_{2}, \omega_{3}$, e.g. with the interpretation "at state $\omega_{i}$ ticket $i$ is the winner" (assuming that there is one and only one winner) and $x=\frac{1}{3}$. Let the atomic proposition $p_{-i}$ be "ticket $i$ is not the winner" so that, for example, $\left\|p_{-1}\right\|=\left\{\omega_{2}, \omega_{3}\right\}$. Then, letting $\mathcal{C}\left(\omega_{i}\right)=\left\{\omega_{1}, \omega_{2}, \omega_{3}\right\}$, for every $i \in\{1,2,3\}$, we have that $\omega_{2} \vDash B p_{-1}$ and $\omega_{2} \vDash B p_{-3}$ but $\omega_{2} \not \forall B\left(p_{-1} \wedge p_{-3}\right)$.

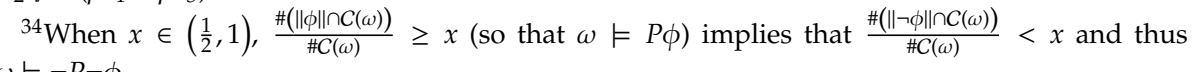
$\omega \vDash \neg P \neg \phi$.
} 
where $S$ is the (possibly empty) collection of axioms from the set $\left\{D_{C}, 4_{C}, 5_{C}\right.$, $T_{C}$ that are postulated for certainty. ${ }^{35}$

3. $x=1$. In this case we get the reduction of belief to certainty: $\omega \vDash B \phi$ if and only $\omega \vDash C \phi .^{36}$

\subsection{Other related literature}

As mentioned in the Introduction, there is a long tradition in several disciplines where the agent is modeled as having two epistemic levels, typically called knowledge and belief. Both notions are taken to be primitives and their relationship is explored either from a syntactic or from a semantic point of view. Our approach is similar, in that we also start with two primitive notions (certainty and plausibility), but in our framework belief is a derived notion. While certainty is modeled in the standard way, by means of a normal syntactic operator (or, semantically, as a binary Kripke relation), plausibility is treated as a more general concept: syntactically it is only required to be a classical operator and semantically it is modeled by means of a neighborhood function. Along somewhat similar lines, Balbiani et al. (2018) investigate a logic that distinguishes the concept of "explicit belief" from the concept of "background knowledge". They use a relational semantics for background knowledge and a neighbourhood semantics for explicit beliefs and discuss axioms that express the relationship between the two concepts. They take the two concepts of background knowledge and explicit belief as primitive notions.

There are several contributions in the literature where belief is a derived notion: derived from knowledge and/or plausibility. In some papers the agent is said to believe $\phi$ if he/she knows $\phi$ to be true in the most plausible states. ${ }^{37}$ We showed in Section 6.2 that belief as truth in the most plausible (or preferred) states is a special case of our framework. In other papers (e.g. Lamarre and Shoham (1994)) plausibility is the only primitive and is used to define both knowledge and belief. Other authors (e.g. Boutilier (1992), Goldszmidt and Pearl (1992)) postulate a preference ordering over possible states to characterize formulas of the form "after learning $\psi$, the agent believes $\phi$ "; this approach is

\footnotetext{
${ }^{35}$ The Lottery Paradox can also be captured in Case 2 (in particular, when $x$ is close to 1 ).

${ }^{36}$ If $\phi$ is true at $100 \%$ of the states accessible from $\omega$ then, at $\omega$, the individual is certain that $\phi$.

${ }^{37}$ For example, Moses and Shoham (1993). However, in that paper plausibility is not defined by an ordering but in terms of a formula, which can be thought of identifying the most plausible states.
} 
linked to the extensive literature on the AGM theory of belief revision (for a survey see Fermé and Hansson $(2011 ; 2018)$ ).

Perhaps the closest paper to ours is Friedman and Halpern (1997). The authors start with two primitive notions: knowledge and plausibility. Knowledge is modeled semantically by a reflexive and Euclidean Kripke relation (and syntactically by an S5 operator), while plausibility is defined as a plausibility measure which allows one to compare any two events in terms of their relative plausibility; thus their framework has more structure than ours since it allows one to make assertions of the form "event $E$ is more plausible than event $F$ ", denoted by $P l(E) \geq P l(F)$. Syntactically, the plausibility measure is represented by a binary modal operator $\hookrightarrow$ and the interpretation of $\phi \hookrightarrow \psi$ is "according to the agent, $\phi$ typically implies $\psi^{\prime \prime}$; the validation rule is as follows: $\omega \vDash \phi \hookrightarrow \psi$ if either $P l(\|\phi\|)=\perp$ or $P l(\|\phi \wedge \psi\|)>P l(\|\phi \wedge \neg \psi\|)$. The authors then define belief as follows: the agent believes $\phi$ if and only if he knows that $\phi$ is more plausible than $\neg \phi: \quad B \phi \leftrightarrow K($ true $\hookrightarrow \phi)$. As we did in Sections 3 and 5, the authors then consider a number of properties defining the interaction between knowledge and plausibility and study how these properties are translated into properties of belief. ${ }^{38}$ It is clear that our framework is "lighter" than theirs, since we model plausibility as a unary modal operator, which is not even required to be a normal operator (that is, it does not correspond to a binary Kripke relation) and we do not impose the S5 logic on the certainty operator. The less structure one imposes, the clearer it is to grasp what is really necessary in order to obtain "desirable" properties of belief (such as positive and negative introspection). ${ }^{39}$

\section{$7 \quad$ Alternative minimal extensions of $\mathbb{L}$}

In this section we consider alternative minimal extensions of logic $\mathbb{L}$ that yield some of the theorems considered in Section 3.

Recall that, by Proposition 6, the schema $(B \phi \rightarrow C B \phi)$ is a theorem of logic $\mathbb{L}+\left\{C P_{1}, C P_{3}, C P_{4}\right\}$. The following proposition shows that one can replace Axiom $\left(C P_{1}\right)$ with $\left(5_{C}\right)$ (negative introspection of certainty) and obtain the same result.

\footnotetext{
${ }^{38}$ Note that the analysis in Friedman and Halpern (1997) goes beyond this, because they also introduce time and use the extended framework to incorporate belief revision and belief update.

${ }^{39}$ For example, we showed that no interaction properties (between certainty and plausibility) at all are needed to obtain consistency of beliefs (Proposition 1).
} 


\section{Proposition 18.}

$$
\mathbb{L}+\left\{\begin{array}{ll}
\left(5_{C}\right) & \neg C \phi \rightarrow C \neg C \phi \\
\left(C P_{3}\right) & \neg P \phi \rightarrow C \neg P \phi \\
\left(C P_{4}\right) & P \phi \rightarrow C P \phi
\end{array}\right\} \vdash B \phi \rightarrow C B \phi
$$

The proof consists of Steps 1-4 in the proof of Proposition 6 while Steps 5-7 are replaced by

$$
\begin{array}{lll}
\text { 5. } & \neg C \neg \phi \rightarrow C \neg C \neg \phi & \text { (Axiom 5C) } \\
\text { 6. } & B \phi \rightarrow(C P \phi \wedge C \neg P \neg \phi \wedge \neg C \neg \phi) & (1,4, \mathrm{PL}) \\
\text { 7. } & B \phi \rightarrow(C P \phi \wedge C \neg P \neg \phi \wedge C \neg C \neg \phi) & (5,6, P L)
\end{array}
$$

step 8 is deleted (it is a copy of the new step 7) and then the proof continues with Steps 9-12 in the proof of Proposition 6, re-numbered as Steps 8-11 (the reference to 8 and 9 in the new step 9 should be replaced by 7 and 8 and the reference 10 and 11 in the last step should be replaced by 9 and 10).

Remark 8. In virtue of Proposition 18 and the proof of Proposition 10, the schema $B \neg B \phi \rightarrow \neg B \phi$ is also a theorem of the logic obtained by replacing Axiom $\left(C P_{1}\right)$ with Axiom (5C) in Proposition $10,{ }^{40}$ that is,

$$
\mathbb{L}+\left\{\begin{array}{ll}
\left(5_{C}\right) & \neg C \phi \rightarrow C \neg C \phi \\
\left(C P_{3}\right) & \neg P \phi \rightarrow C \neg P \phi \\
\left(C P_{4}\right) & P \phi \rightarrow C P \phi
\end{array}\right\} \vdash B \neg B \phi \rightarrow \neg B \phi
$$

Furthermore, the logic $\mathbb{L}+\left\{5_{C}, C P_{3}, C P_{4}\right\}$ is a minimal extension of $\mathbb{L}$ that yields $(B \phi \rightarrow C B \phi)$ and $(B \neg B \phi \rightarrow \neg B \phi)$ as theorems: see Footnotes 42, 50 and 51.

By Proposition 7, the schema $(\neg B \phi \rightarrow C \neg B \phi)$ is a theorem of logic $\mathbb{L}+$ $\left\{C P_{2}, C P_{3}, C P_{4}\right\}$. The following proposition shows that one can replace Axiom $\left(C P_{2}\right)$ with $\left(4_{C}\right)$ (positive introspection of certainty) and obtain the same result.

\section{Proposition 19.}

$$
\mathbb{L}+\left\{\begin{array}{ll}
\left(4_{C}\right) & C \phi \rightarrow C C \phi \\
\left(C P_{3}\right) & \neg P \phi \rightarrow C \neg P \phi \\
\left(C P_{4}\right) & P \phi \rightarrow C P \phi
\end{array}\right\} \vdash \neg B \phi \rightarrow C \neg B \phi
$$

Proof.

\footnotetext{
${ }^{40}$ In step 3 of the proof of Proposition 10 the call would be to Proposition 18 instead of Proposition 6.
} 
1. $C \neg \phi \rightarrow C C \neg \phi$

(Axiom $4_{C}$ )

2. $\neg C C \neg \phi \rightarrow \neg C \neg \phi$

3. $\neg P \phi \rightarrow C \neg P \phi$

(Axiom $\mathrm{CP}_{3}$ )

4. $\neg C \neg P \phi \rightarrow P \phi$

$(3, \mathrm{PL})$

5. $\quad P \neg \phi \rightarrow C P \neg \phi$

(Axiom $\mathrm{CP}_{4}$ )

6. $\neg C P \neg \phi \rightarrow \neg P \neg \phi$

7. $(\neg C C \neg \phi \wedge \neg C \neg P \phi \wedge \neg C P \neg \phi) \rightarrow(\neg C \neg \phi \wedge P \phi \wedge \neg P \neg \phi)$

8. $\quad(\neg C \neg \phi \wedge P \phi \wedge \neg P \neg \phi) \leftrightarrow B \phi$

9. $\quad(\neg C C \neg \phi \wedge \neg C \neg P \phi \wedge \neg C P \neg \phi) \rightarrow B \phi$

10. $\neg C \neg(\neg C \neg \phi \wedge P \phi \wedge \neg P \neg \phi) \leftrightarrow \neg C \neg B \phi$

11. $\neg C \neg(\neg C \neg \phi \wedge P \phi \wedge \neg P \neg \phi) \rightarrow(\neg C C \neg \phi \wedge \neg C \neg P \phi \wedge \neg C P \neg \phi)$

(Remark 3)

12. $\neg C \neg B \phi \rightarrow B \phi$

13. $\neg B \phi \rightarrow C \neg B \phi$.

Remark 9. In virtue of Proposition 19 and the proof of Proposition 9, the schema $B B \phi \rightarrow B \phi$ is also a theorem of the logic obtained by replacing Axiom $\left(C_{2}\right)$ with Axiom $\left(4_{C}\right)$ in Proposition 9, ${ }^{41}$ that is,

$$
\mathbb{L}+\left\{\begin{array}{ll}
\left(4_{C}\right) & \neg C \phi \rightarrow C \neg C \phi \\
\left(C P_{3}\right) & \neg P \phi \rightarrow C \neg P \phi \\
\left(C P_{4}\right) & P \phi \rightarrow C P \phi
\end{array}\right\} \vdash B B \phi \rightarrow B \phi
$$

Furthermore, the logic $\mathbb{L}+\left\{4_{C}, C P_{3}, C P_{4}\right\}$ is a minimal extension of $\mathbb{L}$ that yields $(\neg B \phi \rightarrow C \neg B \phi)$ and $(B B \phi \rightarrow B \phi)$ as theorems: see Footnotes 43,47 and 48 .

On the other hand, by inspecting the proof of minimality of the logic of Proposition 8 , one can see that positive and negative introspection of belief are not theorems of a logic obtained by adding Axioms $\left(4_{C}\right)$ and $\left(5_{C}\right)$ to $\mathbb{L}$ and any proper subset of the set of axioms $\left\{C P_{1}, C P_{2}, C P_{3}, C P_{4}\right\}$.

\footnotetext{
${ }^{41}$ In step 1 of the proof of Proposition 9 the call would be to Proposition 19 instead of Proposition 7
} 


\section{Conclusion}

From the two primitive notions of certainty and plausibility we derived belief as "maximally plausible possibility": the agent believes $\phi$ if (1) she cannot rule out $\phi,(2)$ she judges $\phi$ to be plausible and (3) she does not judge $\neg \phi$ to be plausible. We then considered interaction properties between certainty and plausibility and studied how these properties translate into properties of belief (such as positive and negative introspection and their converses). Our purpose was to identify minimal logics that would yield those properties of belief. In order to do so we started with a basic logic where certainty is modeled as a normal operator and plausibility as a weaker operator (a classical operator) and then added as few axioms as possible (concerning certainty and the interaction between certainty and plausibility) to obtain various properties of beliefs. The analysis was carried out syntactically, but in Section 4 we introduced the semantics in order to prove the minimality of the various logics considered. The semantics for certainty was specified in terms of a standard binary Kripke relation, while plausibility was represented by a neighborhood function. In Section 6 we considered a number of possible interpretations of plausibility (thereby establishing a link to some of the literature) and identified the minimal logic associated with each interpretation. In future work we plan to apply (a multi-agent version of) the framework introduced in this paper to a qualitative analysis of game theoretic notions.

\section{A Proof of Proposition 13}

The proof for Proposition 5 was given in Section 4 . Thus we only need to give a proof of minimality for Propositions 6-10.

Proof of minimality for Proposition 6. We need to prove that the schema $B \phi \rightarrow C B \phi$ is not a theorem of any sub-logic of $\mathbb{L}+\left\{\begin{array}{ll}\left(C P_{1}\right) & C \phi \rightarrow P \phi \\ \left(C P_{3}\right) & \neg P \phi \rightarrow C \neg P \phi \\ \left(C P_{4}\right) & P \phi \rightarrow C P \phi\end{array}\right\}$.

$\backslash C P_{1}$ Let $\Omega=\{\alpha, \beta\}, C(\alpha)=\{\alpha, \beta\}, C(\beta)=\{\beta\}, \mathcal{P}(\alpha)=\mathcal{P}(\beta)=\{\{\alpha\}, \Omega\}$. This frame satisfies Properties $\left(\mathrm{F}_{\mathrm{CP}_{3}}\right)$ and $\left(\mathrm{F}_{C P_{4}}\right)$ (and thus validates Axioms $\left(C P_{3}\right)$ and $\left.\left(C P_{4}\right)\right)$ but violates Property $\left(\mathrm{F}_{C P_{1}}\right)$ since $C(\beta) \subseteq\{\beta\}$ but $\{\beta\} \notin$ $\mathcal{P}(\beta) .{ }^{42}$ Let $p$ be an atomic formula and construct a model based on this

${ }^{42}$ Note that $C$ is transitive but not Euclidean. Indeed, as implied by Proposition 18, it cannot 
frame where $\|p\|=\{\alpha\}$. Then $\|B p\|=\{\alpha\}(\beta \not \forall B p$ since $C(\beta) \cap\|p\|=\varnothing)$ and thus $\alpha \vDash B p$ but (since $\beta \in C(\alpha)) \alpha \not \models C B p$.

$\backslash C P_{3}$ Let $\Omega=\{\alpha, \beta\}, C(\alpha)=C(\beta)=\{\beta\}, \mathcal{P}(\alpha)=\{\{\beta\}, \Omega\}, \mathcal{P}(\beta)=\{\{\alpha\},\{\beta\}, \Omega\}$. This frame satisfies Properties $\left(\mathrm{F}_{C P_{1}}\right)$ and $\left(\mathrm{F}_{C P_{4}}\right)$ (and thus validates Axioms $\left(C P_{1}\right)$ and $\left.\left(C P_{4}\right)\right)$ but violates Property $\left(\mathrm{F}_{C P_{3}}\right)$ since $\beta \in C(\alpha)$ but $\mathcal{P}(\beta) \nsubseteq \mathcal{P}(\alpha)$. Let $p$ be an atomic formula and construct a model based on this frame where $\|p\|=\{\beta\}$. Then $\|B p\|=\{\alpha\}(\beta \not \forall B p$ since $\Omega \backslash\|p\|=\{\alpha\} \in \mathcal{P}(\beta)$ and thus $\beta \not \models \neg \neg p)$ so that $\alpha \vDash B p$ but $\alpha \not \forall C B p$.

$\backslash C P_{4}$ Let $\Omega=\{\alpha, \beta, \gamma\}, C(\alpha)=C(\beta)=C(\gamma)=\{\beta, \gamma\}, \mathcal{P}(\alpha)=\{\{\beta\},\{\beta, \gamma\}, \Omega\}, \mathcal{P}(\beta)=$ $\mathcal{P}(\gamma)=\{\{\beta, \gamma\}, \Omega\}$. This frame satisfies Properties $\left(\mathrm{F}_{C P_{1}}\right)$ and $\left(\mathrm{F}_{C P_{3}}\right)$ (and thus validates Axioms $\left(C P_{1}\right)$ and $\left.\left(C_{3}\right)\right)$ but violates Property $\left(\mathrm{F}_{C P_{4}}\right)$ since $\beta \in C(\alpha)$ but $\mathcal{P}(\alpha) \nsubseteq \mathcal{P}(\beta)$. Let $p$ be an atomic formula and construct a model based on this frame where $\|p\|=\{\beta\}$. Then $\|B p\|=\{\alpha\}(\beta \not \forall B p$ since $\|p\| \notin \mathcal{P}(\beta)$ and thus $\beta \not P P$ and the same is true of $\gamma$ ) so that $\alpha=B p$ but $\alpha \not \models C B p$.

Proof of minimality for Proposition 7. We need to prove that the schema $\neg B \phi \rightarrow C \neg B \phi$ is not a theorem of any sub-logic of $\mathbb{L}+\left\{\begin{array}{ll}\left(C_{2}\right) & C \phi \rightarrow \neg P \neg \phi \\ \left(C_{3}\right) & \neg P \phi \rightarrow C \neg P \phi \\ \left(C P_{4}\right) & P \phi \rightarrow C P \phi\end{array}\right\}$.

$\backslash C P_{2}$ Let $\Omega=\{\alpha, \beta, \gamma\}, \mathcal{C}(\alpha)=\{\beta\}, C(\beta)=C(\gamma)=\{\beta, \gamma\}, \mathcal{P}(\alpha)=\mathcal{P}(\beta)=\mathcal{P}(\gamma)=$ $\{\{\gamma\}, \Omega\}$. This frame satisfies Properties $\left(\mathrm{F}_{\mathrm{CP}_{3}}\right)$ and $\left(\mathrm{F}_{C P_{4}}\right)$ (and thus validates Axioms $\left(\mathrm{CP}_{3}\right)$ and $\left.\left(\mathrm{CP}_{4}\right)\right)$ but violates Property $\left(\mathrm{F}_{C P_{2}}\right)$ since $C(\alpha) \subseteq$ $\{\alpha, \beta\}$ but $(\Omega \backslash\{\alpha, \beta\})=\{\gamma\} \in \mathcal{P}(\alpha)$. Let $p$ be an atomic formula and construct a model based on this frame where $\|p\|=\{\gamma\}$. Then $\|B p\|=\{\beta, \gamma\}$ $(\alpha \not \forall B p$ because $C(\alpha) \cap\|p\|=\varnothing)$. Thus $\alpha=(\neg B p \wedge \neg C \neg B p){ }^{43}$

$\backslash C P_{3}$ Let $\Omega=\{\alpha, \beta\}, C(\alpha)=C(\beta)=\{\beta\}, \mathcal{P}(\alpha)=\{\Omega\}, \mathcal{P}(\beta)=\{\{\beta\}, \Omega\}$. This frame satisfies Properties $\left(\mathrm{F}_{\mathrm{CP}_{2}}\right)$ and $\left(\mathrm{F}_{\mathrm{CP}_{4}}\right)$ (and thus validates Axioms $\left(\mathrm{CP}_{2}\right)$ and $\left.\left(C_{4}\right)\right)$ but violates Property $\left(\mathrm{F}_{C P_{3}}\right)$ since $\beta \in C(\alpha)$ but $\mathcal{P}(\beta) \nsubseteq \mathcal{P}(\alpha)$. Let $p$ be an atomic formula and construct a model based on this frame where $\|p\|=\{\beta\}$. Then $\|B p\|=\{\beta\}(\alpha \not \neq B p$ since $\|p\| \notin \mathcal{P}(\alpha))$ and thus $\alpha \vDash(\neg B p \wedge \neg C \neg B p)$.

be Euclidean. Note also that the proof given for Proposition 6 is also a proof of minimality for the logic of Proposition 18 since in the remaining two cases $C$ is in fact Euclidean.

${ }^{43}$ Note that $C$ is not transitive. Indeed, as implied by Proposition 19, it cannot be transitive. Note also that the proof given for Proposition 7 is also a proof of minimality for the logic of Proposition 19 since in the remaining two cases $C$ is in fact transitive. 
$\backslash C P_{4}$ Let $\Omega=\{\alpha, \beta, \gamma\}, \mathcal{C}(\alpha)=\{\beta, \gamma\}, \mathcal{C}(\beta)=C(\gamma)=\{\gamma\}, \mathcal{P}(\alpha)=\{\{\gamma\},\{\alpha, \beta\}, \Omega\}$, $\mathcal{P}(\beta)=\mathcal{P}(\gamma)=\{\{\gamma\}, \Omega\}$. This frame satisfies Properties $\left(\mathrm{F}_{C P_{2}}\right)$ and $\left(\mathrm{F}_{C P_{3}}\right)$ (and thus validates Axioms $\left(\mathrm{CP}_{2}\right)$ and $\left.\left(\mathrm{CP}_{3}\right)\right)$ but violates Property $\left(\mathrm{F}_{\mathrm{CP}}\right)$ since $\beta \in C(\alpha)$ but $\mathcal{P}(\alpha) \nsubseteq \mathcal{P}(\beta)$. Let $p$ be an atomic formula and construct a model based on this frame where $\|p\|=\{\gamma\}$. Then $\|B p\|=\{\beta, \gamma\}(\alpha \not \forall B p$ since $\Omega \backslash\|p\|=\{\alpha, \beta\} \in \mathcal{P}(\alpha)$ so that $\alpha \not \models \neg P \neg p)$. Thus $\alpha \vDash(\neg B p \wedge \neg C \neg B p)$.

Proof of minimality for Proposition 8.

First we prove that the schema $B \phi \rightarrow B B \phi$ is not a theorem of any sub-logic of $\mathbb{L}+\left\{\begin{array}{ll}\left(C P_{1}\right) & C \phi \rightarrow P \phi \\ \left(C P_{2}\right) & C \phi \rightarrow \neg P \neg \phi \\ \left(C P_{3}\right) & \neg P \phi \rightarrow C \neg P \phi \\ \left(C P_{4}\right) & P \phi \rightarrow C P \phi\end{array}\right\}$.

$\backslash C P_{1}$ Let $\Omega=\{\alpha, \beta, \gamma\}, C(\alpha)=C(\beta)=\{\beta\}, C(\gamma)=\{\gamma\}, \mathcal{P}(\alpha)=\mathcal{P}(\beta)=\{\{\beta\}, \Omega\}$, $\mathcal{P}(\gamma)=\{\{\gamma\},\{\alpha, \gamma\},\{\beta, \gamma\}, \Omega\}$. This frame satisfies Properties $\left(\mathrm{F}_{C_{2}}\right),\left(\mathrm{F}_{C_{3}}\right)$ and $\left(\mathrm{F}_{C_{4}}\right)$ (and thus validates Axioms $\left(C P_{2}\right),\left(C P_{3}\right)$ and $\left.\left(C P_{4}\right)\right)^{44}$ but violates Property $\left(\mathrm{F}_{C P_{1}}\right)$ since $C(\alpha) \subseteq\{\beta, \gamma\}$ but $\{\beta, \gamma\} \notin \mathcal{P}(\alpha)$. Let $p$ be an atomic formula and construct a model based on this frame where $\|p\|=$ $\{\beta\}$. Then $\|B p\|=\{\alpha, \beta\}$ so that $\alpha \models B p$ but $\alpha \not \models B B p$ since $\|B p\| \notin \mathcal{P}(\alpha)$.

$\backslash C_{2}$ Let $\Omega=\{\alpha, \beta, \gamma\}, \mathcal{C}(\alpha)=\mathcal{C}(\beta)=\{\beta\}, \mathcal{C}(\gamma)=\{\gamma\}, \mathcal{P}(\alpha)=\mathcal{P}(\beta)=$ $\{\{\beta\},\{\gamma\},\{\alpha, \beta\},\{\beta, \gamma\}, \Omega\}, \mathcal{P}(\gamma)=\{\{\gamma\},\{\alpha, \gamma\},\{\beta, \gamma\}, \Omega\}$. This frame satisfies Properties $\left(\mathrm{F}_{C P_{1}}\right),\left(\mathrm{F}_{C P_{3}}\right)$ and $\left(\mathrm{F}_{C P_{4}}\right)$ (and thus validates Axioms $\left(C P_{1}\right)$, $\left(C P_{3}\right)$ and $\left.\left(C P_{4}\right)\right)^{45}$ but violates Property $\left(\mathrm{F}_{C P_{2}}\right)$ since $C(\alpha) \subseteq\{\alpha, \beta\}$ and $\Omega \backslash\{\alpha, \beta\}=\{\gamma\} \in \mathcal{P}(\alpha)$. Let $p$ be an atomic formula and construct a model based on this frame where $\|p\|=\{\beta\}$. Then $\|B p\|=\{\alpha, \beta\}$ so that $\alpha=B p$ but $\alpha \not \models B B p$ since $\alpha \not \forall \neg P \neg B p$ (because $\|\neg B p\|=\{\gamma\} \in \mathcal{P}(\alpha)$ ).

$\backslash C P_{3}$ Let $\Omega=\{\alpha, \beta, \gamma, \delta\}, C(\alpha)=\{\alpha, \beta\}, C(\beta)=C(\gamma)=C(\delta)=\{\beta, \gamma, \delta\}$, $\mathcal{P}(\alpha)=\{\{\alpha, \beta\},\{\alpha, \beta, \gamma\},\{\alpha, \beta, \delta\}, \Omega\}, \quad \mathcal{P}(\beta)=\mathcal{P}(\gamma)=\mathcal{P}(\delta)=$ $\{\{\alpha, \beta\},\{\gamma, \delta\},\{\alpha, \beta, \gamma\},\{\alpha, \beta, \delta\},\{\beta, \gamma, \delta\}, \Omega\}$. This frame satisfies Properties $\left(\mathrm{F}_{C P_{1}}\right),\left(\mathrm{F}_{C P_{2}}\right)$ and $\left(\mathrm{F}_{C P_{4}}\right)$ (and thus validates Axioms $\left(C P_{1}\right),\left(C P_{2}\right)$ and $\left.\left(C P_{4}\right)\right)$ but violates Property $\left(\mathrm{F}_{C_{3}}\right)$ since $\beta \in C(\alpha)$ but $\mathcal{P}(\beta) \nsubseteq \mathcal{P}(\alpha)$. Let $p$ be an atomic formula and construct a model based on this frame where $\|p\|=\{\alpha, \beta\}$. Then $\|B p\|=\{\alpha\}(\beta \notin\|B p\|$ because $\Omega \backslash\|p\|=\{\gamma, \delta\} \in \mathcal{P}(\beta)$ and the same is true of $\gamma$ and $\delta$ ) so that $\alpha \models B p$ but $\alpha \not \models B B p$ since $\|B p\| \notin \mathcal{P}(\alpha)$.

\footnotetext{
${ }^{44}$ Note also that $C$ is transitive and Euclidean and thus validates Axioms $\left(4_{C}\right)$ and $\left(5_{C}\right)$.
}

${ }^{45}$ Note also that $C$ is transitive and Euclidean and thus validates Axioms $\left(4_{C}\right)$ and $\left(5_{C}\right)$. 
$\backslash C P_{4}$ Let $\Omega=\{\alpha, \beta, \gamma\}, C(\alpha)=C(\beta)=C(\gamma)=\{\beta, \gamma\},{ }^{46} \mathcal{P}(\alpha)=\{\{\beta\},\{\beta, \gamma\}, \Omega\}$, $\mathcal{P}(\beta)=\mathcal{P}(\gamma)=\{\{\beta, \gamma\}, \Omega\}$. This frame satisfies Properties $\left(\mathrm{F}_{C P_{1}}\right),\left(\mathrm{F}_{C P_{2}}\right)$ and $\left(\mathrm{F}_{C P_{3}}\right)$ (and thus validates Axioms $\left(C P_{1}\right),\left(C P_{2}\right)$ and $\left.\left(C P_{3}\right)\right)$ but violates Property $\left(\mathrm{F}_{\mathrm{CP}_{4}}\right)$ since $\beta \in \mathcal{C}(\alpha)$ but $\mathcal{P}(\alpha) \nsubseteq \mathscr{P}(\beta)$. Let $p$ be an atomic formula and construct a model based on this frame where $\|p\|=\{\beta\}$. Then $\|B p\|=\{\alpha\}(\beta \notin\|B p\|$ because $\|p\| \notin \mathcal{P}(\beta)$ and the same is true of $\gamma)$ so that $\alpha \models B p$ but $\alpha \not \models B B p$ since $C(\alpha) \cap\|B p\|=\varnothing$.

Next we prove that the schema $\neg B \phi \rightarrow B \neg B \phi$ is not a theorem of any sub-logic of $\mathbb{L}+\left\{\begin{array}{ll}\left(C P_{1}\right) & C \phi \rightarrow P \phi \\ \left(C P_{2}\right) & C \phi \rightarrow \neg P \neg \phi \\ \left(C P_{3}\right) & \neg P \phi \rightarrow C \neg P \phi \\ \left(C P_{4}\right) & P \phi \rightarrow C P \phi\end{array}\right\}$. Note that in all the frames considered below, $C$ is transitive and Euclidean and thus validates Axioms $\left(4_{C}\right)$ and $\left(5_{C}\right)$.

$\backslash C P_{1}$ Let $\Omega=\{\alpha, \beta, \gamma\}, \mathcal{C}(\alpha)=\mathcal{C}(\beta)=\{\beta\}, \mathcal{C}(\gamma)=\{\gamma\}, \mathcal{P}(\alpha)=\mathcal{P}(\beta)=\{\Omega\}, \mathcal{P}(\gamma)=$ $\{\{\beta, \gamma\}, \Omega\}$. This frame satisfies Properties $\left(\mathrm{F}_{C P_{2}}\right),\left(\mathrm{F}_{C P_{3}}\right)$ and $\left(\mathrm{F}_{C P_{4}}\right)$ (and thus validates Axioms $\left(C P_{2}\right),\left(C P_{3}\right)$ and $\left.\left(C P_{4}\right)\right)$ but violates Property $\left(\mathrm{F}_{C P_{1}}\right)$ since $C(\alpha) \subseteq\{\beta\}$ but $\{\beta\} \notin \mathcal{P}(\alpha)$. Let $p$ be an atomic formula and construct a model based on this frame where $\|p\|=\{\beta, \gamma\}$. Then $\|B p\|=\{\gamma\}(\alpha \notin\|B p\|$ because $\|p\| \notin \mathcal{P}(\alpha)$ and the same is true of $\beta)$. Thus $\alpha \models \neg B p$ but $\alpha \forall B \neg B p$ because $\|\neg B p\|=\Omega \backslash\|B p\|=\{\alpha, \beta\} \notin \mathcal{P}(\alpha)$.

$\backslash C P_{2}$ Let $\Omega=\{\alpha, \beta, \gamma\}, C(\alpha)=C(\beta)=\{\beta\}, C(\gamma)=\{\gamma\}, \mathcal{P}(\alpha)=\mathcal{P}(\beta)=$ $\{\{\alpha\},\{\beta\},\{\gamma\},\{\alpha, \beta\},\{\beta, \gamma\}, \Omega\}, \mathcal{P}(\gamma)=\{\{\gamma\},\{\alpha, \gamma\},\{\beta, \gamma\}, \Omega\}$. This frame satisfies Properties $\left(\mathrm{F}_{C P_{1}}\right),\left(\mathrm{F}_{\mathrm{CP}_{3}}\right)$ and $\left(\mathrm{F}_{C P_{4}}\right)$ (and thus validates Axioms $\left(C P_{1}\right),\left(C P_{3}\right)$ and $\left.\left(C P_{4}\right)\right)$ but violates Property $\left(\mathrm{F}_{C P_{2}}\right)$ since $C(\alpha) \subseteq\{\beta, \gamma\}$ and $\Omega \backslash\{\beta, \gamma\}=\{\alpha\} \in \mathcal{P}(\alpha)$. Let $p$ be an atomic formula and construct a model based on this frame where $\|p\|=\{\beta, \gamma\}$. Then $\|B p\|=\{\gamma\}(\alpha \notin\|B p\|$ because $\Omega \backslash\|p\| \in \mathcal{P}(\alpha)$ and the same is true of $\beta$ ). Thus $\alpha \models \neg B p$ but $\alpha \not \forall B \neg B p$ because $\|\neg B p\|=\Omega \backslash\|B p\|=\{\alpha, \beta\}$ and $\Omega \backslash\|\neg B p\|=\{\gamma\} \in \mathcal{P}(\alpha)$.

$\backslash C P_{3}$ Let $\Omega=\{\alpha, \beta, \gamma\}, \mathcal{C}(\alpha)=\mathcal{C}(\beta)=\mathcal{C}(\gamma)=\{\beta, \gamma\}, \mathcal{P}(\alpha)=\{\{\beta, \gamma\}, \Omega\}, \mathcal{P}(\beta)=$ $\mathcal{P}(\gamma)=\{\{\alpha, \beta\},\{\beta, \gamma\}, \Omega\}$. This frame satisfies Properties $\left(\mathrm{F}_{C P_{1}}\right),\left(\mathrm{F}_{C P_{2}}\right)$ and $\left(\mathrm{F}_{C P_{4}}\right)$ (and thus validates Axioms $\left(C P_{1}\right),\left(C P_{2}\right)$ and $\left(C P_{4}\right)$ ) but violates Property $\left(\mathrm{F}_{\mathrm{CP}_{3}}\right)$ since $\beta \in \mathcal{C}(\alpha)$ but $\mathcal{P}(\beta) \nsubseteq \mathcal{P}(\alpha)$. Let $p$ be an atomic formula and construct a model based on this frame where $\|p\|=\{\alpha, \beta\}$. Then $\|B p\|=\{\beta, \gamma\}(\alpha \not \models B p$ since $\|p\| \notin \mathcal{P}(\alpha))$. Thus $\alpha \models \neg B p$ but $\alpha \not \forall B \neg B p$ because $\|\neg B p\|=\Omega \backslash\|B p\|=\{\alpha\}$ and $C(\alpha) \cap\{\alpha\}=\varnothing$.

\footnotetext{
${ }^{46}$ Note that $C$ is transitive and Euclidean and thus validates Axioms $\left(4_{C}\right)$ and $\left(5_{C}\right)$.
} 
$\backslash C P_{4}$ Let $\Omega=\{\alpha, \beta, \gamma\}, \mathcal{C}(\alpha)=C(\beta)=C(\gamma)=\{\beta, \gamma\}, \mathcal{P}(\alpha)=\{\{\gamma\},\{\alpha, \beta\},\{\beta, \gamma\}, \Omega\}$, $\mathcal{P}(\beta)=\mathcal{P}(\gamma)=\{\{\alpha, \beta\},\{\beta, \gamma\}, \Omega\}$. This frame satisfies Properties $\left(\mathrm{F}_{C P_{1}}\right)$, $\left(\mathrm{F}_{C P_{2}}\right)$ and $\left(\mathrm{F}_{C P_{3}}\right)$ (and thus validates Axioms $\left(C P_{1}\right),\left(C P_{2}\right)$ and $\left(C P_{3}\right)$ ) but violates Property $\left(\mathrm{F}_{C P_{4}}\right)$ since $\beta \in C(\alpha)$ but $\mathcal{P}(\alpha) \nsubseteq \mathcal{P}(\beta)$. Let $p$ be an atomic formula and construct a model based on this frame where $\|p\|=\{\alpha, \beta\}$. Then $\|B p\|=\{\beta, \gamma\}(\alpha \not \neq B p$ since $\Omega \backslash\|p\|=\{\gamma\} \in \mathcal{P}(\alpha))$. Thus $\alpha \vDash \neg B p$ but $\alpha \not \forall B \neg B p$ because $\|\neg B p\|=\Omega \backslash\|B p\|=\{\alpha\}$ and $C(\alpha) \cap\{\alpha\}=\varnothing$.

Proof of minimality for Proposition 9. We need to prove that the schema $B B \phi \rightarrow B \phi$ is not a theorem of any sub-logic of $\mathbb{L}+\left\{\begin{array}{ll}\left(C P_{2}\right) & C \phi \rightarrow \neg P \neg \phi \\ \left(C P_{3}\right) & \neg P \phi \rightarrow C \neg P \phi \\ \left(C P_{4}\right) & P \phi \rightarrow C P \phi\end{array}\right\}$.

$\backslash C P_{2}$ Let $\Omega=\{\alpha, \beta, \gamma\}, \mathcal{C}(\alpha)=\{\beta\}, \mathcal{C}(\beta)=\mathcal{C}(\gamma)=\{\gamma\}, \mathcal{P}(\alpha)=\mathcal{P}(\beta)=\mathcal{P}(\gamma)=$ $\{\{\gamma\},\{\alpha, \gamma\},\{\beta, \gamma\}, \Omega\}$. This frame satisfies Properties $\left(\mathrm{F}_{C P_{3}}\right)$ and $\left(\mathrm{F}_{C P_{4}}\right)$ (and thus validates Axioms $\left(\mathrm{CP}_{3}\right)$ and $\left.\left(\mathrm{CP}_{4}\right)\right)$ but violates Property $\left(\mathrm{F}_{C P_{2}}\right)$ since $C(\alpha) \subseteq\{\beta\}$ but $\Omega \backslash\{\beta\}=\{\alpha, \gamma\} \in \mathcal{P}(\alpha)$. Let $p$ be an atomic formula and construct a model based on this frame where $\|p\|=\{\alpha, \gamma\}$. Then $\|B p\|=\{\beta, \gamma\}(\alpha \notin\|B p\|$ because $C(\alpha) \cap\|p\|=\varnothing)$ so that $\alpha \models B B p$ but $\alpha \not \models B p$.

$\backslash C P_{3}$ Let $\Omega=\{\alpha, \beta, \gamma\}, C(\alpha)=\{\beta, \gamma\}, C(\beta)=C(\gamma)=\{\gamma\},{ }^{47} \mathcal{P}(\alpha)=\{\{\beta, \gamma\}, \Omega\}$, $\mathcal{P}(\beta)=\mathcal{P}(\gamma)=\{\{\gamma\},\{\alpha, \gamma\},\{\beta, \gamma\}, \Omega\}$. This frame satisfies Properties $\left(\mathrm{F}_{\mathrm{CP}_{2}}\right)$ and $\left(\mathrm{F}_{C P_{4}}\right)$ (and thus validates Axioms $\left(C p_{2}\right)$ and $\left(C P_{4}\right)$ ) but violates Property $\left(C P_{3}\right)$ since $\beta \in \mathcal{P}(\alpha)$ but $\mathcal{P}(\beta) \nsubseteq \mathcal{P}(\alpha)$. Let $p$ be an atomic formula and construct a model based on this frame where $\|p\|=\{\alpha, \gamma\}$. Then $\|B p\|=\{\beta, \gamma\}$ so that $\alpha \models B B p$ but $\alpha \not \models B p$ since $\|p\| \notin \mathcal{P}(\alpha)$.

$\backslash C P_{4}$ Let $\Omega=\{\alpha, \beta, \gamma\}, C(\alpha)=\{\beta, \gamma\}, C(\beta)=C(\gamma)=\{\gamma\},{ }^{48}$ $\mathcal{P}(\alpha)=\{\{\beta\},\{\alpha, \gamma\},\{\beta, \gamma\}, \Omega\}, \quad \mathcal{P}(\beta)=\mathcal{P}(\gamma)=\{\{\alpha, \gamma\},\{\beta, \gamma\}, \Omega\}$. This frame satisfies Properties $\left(\mathrm{F}_{C P_{2}}\right)$ and $\left(\mathrm{F}_{C P_{3}}\right)$ (and thus validates $\mathrm{Ax}$ ioms $\left(C P_{2}\right)$ and $\left.\left(C P_{3}\right)\right)$ but violates Property $\left(C P_{4}\right)$ since $\beta \in \mathcal{P}(\alpha)$ but $\mathcal{P}(\alpha) \nsubseteq \mathcal{P}(\beta)$. Let $p$ be an atomic formula and construct a model based on this frame where $\|p\|=\{\alpha, \gamma\}$. Then $\|B p\|=\{\beta, \gamma\}(\alpha \notin\|B p\|$ because $\Omega \backslash\|p\|=\{\beta\} \in \mathcal{P}(\alpha))$ so that $\alpha \models B B p$ but $\alpha \not \models B p$.

${ }^{47}$ Note that $C$ is transitive.

${ }^{48}$ Note that $C$ is transitive. 
Proof of minimality for Proposition 10. We need to prove that the schema $B \neg B \phi \rightarrow \neg B \phi$ is not a theorem of any sub-logic of $\mathbb{L}+\left\{\begin{array}{ll}\left(C P_{1}\right) & C \phi \rightarrow P \phi \\ \left(C P_{3}\right) & \neg P \phi \rightarrow C \neg P \phi \\ \left(C P_{4}\right) & P \phi \rightarrow C P \phi\end{array}\right\}$.

$\backslash C P_{1}$ Let $\Omega=\{\alpha, \beta, \gamma\}, C(\alpha)=\{\beta, \gamma\}, C(\beta)=\mathcal{C}(\gamma)=\{\gamma\},{ }^{49} \mathcal{P}(\alpha)=\mathcal{P}(\beta)=$ $\mathcal{P}(\gamma)=\{\{\alpha, \beta\},\{\beta, \gamma\}, \Omega\}$. This frame satisfies Properties $\left(\mathrm{F}_{C P_{3}}\right)$ and $\left(\mathrm{F}_{C P_{4}}\right)$ (and thus validates Axioms $\left(C P_{3}\right)$ and $\left.\left(C P_{4}\right)\right)$ but violates Property $\left(\mathrm{F}_{C P_{1}}\right)$ since $C(\beta) \subseteq\{\gamma\}$ but $\{\gamma\} \notin \mathcal{P}(\beta)$. Let $p$ be an atomic formula and construct a model based on this frame where $\|p\|=\{\alpha, \beta\}$. Then $\|B p\|=\{\alpha\}$ $(\beta \notin\|B p\|$ because $C(\beta) \cap\|p\|=\varnothing$ and the same is true of $\gamma$ ) so that $\|\neg B p\|=\Omega \backslash\|B p\|=\{\beta, \gamma\}$ and thus $\alpha \models(B \neg B p \wedge B p)$.

$\backslash C P_{3}$ Let $\Omega=\{\alpha, \beta, \gamma\}, C(\alpha)=C(\beta)=C(\gamma)=\{\beta, \gamma\},{ }^{50} \mathcal{P}(\alpha)=\{\{\alpha, \beta\},\{\beta, \gamma\}, \Omega\}$, $\mathcal{P}(\beta)=\mathcal{P}(\gamma)=\{\{\gamma\},\{\alpha, \beta\},\{\beta, \gamma\}, \Omega\}$. This frame satisfies Properties $\left(\mathrm{F}_{C P_{1}}\right)$ and $\left(\mathrm{F}_{C P_{4}}\right)$ (and thus validates Axioms $\left(C P_{1}\right)$ and $\left(C P_{4}\right)$ ) but violates Property $\left(\mathrm{F}_{\mathrm{CP}_{3}}\right)$ since $\beta \in C(\alpha)$ but $\mathcal{P}(\beta) \nsubseteq \mathcal{P}(\alpha)$. Let $p$ be an atomic formula and construct a model based on this frame where $\|p\|=\{\alpha, \beta\}$. Then $\|B p\|=\{\alpha\}(\beta \notin\|B p\|$ because $\Omega \backslash\|p\|=\{\gamma\} \in \mathcal{P}(\beta)$ and the same is true of $\gamma)$ so that $\|\neg B p\|=\{\beta, \gamma\}$ and thus $\alpha \vDash(B \neg B p \wedge B p)$.

$\backslash C P_{4}$ Let $\Omega=\{\alpha, \beta, \gamma\}, C(\alpha)=C(\beta)=C(\gamma)=\{\beta, \gamma\},{ }^{51} \mathcal{P}(\alpha)=\{\{\alpha, \beta\},\{\beta, \gamma\}, \Omega\}$, $\mathcal{P}(\beta)=\mathcal{P}(\gamma)=\{\{\beta, \gamma\}, \Omega\}$. This frame satisfies Properties $\left(\mathrm{F}_{C P_{1}}\right)$ and $\left(\mathrm{F}_{C P_{3}}\right)$ (and thus validates Axioms $\left(C P_{1}\right)$ and $\left(C P_{3}\right)$ ) but violates Property $\left(\mathrm{F}_{C P_{4}}\right)$ since $\beta \in C(\alpha)$ but $\mathcal{P}(\alpha) \nsubseteq \mathcal{P}(\beta)$. Let $p$ be an atomic formula and construct a model based on this frame where $\|p\|=\{\alpha, \beta\}$. Then $\|B p\|=\{\alpha\}(\beta \notin\|B p\|$ because $\|p\| \notin \mathcal{P}(\beta)$ and the same is true of $\gamma)$ so that $\|\neg B p\|=\{\beta, \gamma\}$ and thus $\alpha \models(B \neg B p \wedge B p)$.

\section{References}

R. Aumann. Agreeing to disagree. The Annals of Statistics, 4:1236-1239, 1976.

R. Aumann. Interactive epistemology II: Probability. International Journal of Game Theory, 28:301-314, 1999.

\footnotetext{
${ }^{49}$ Note that $C$ is transitive but not Euclidean; indeed, it cannot be Euclidean because of Remark 8 .

${ }^{50}$ Note that $C$ is transitive and Euclidean.

${ }^{51}$ Note that $C$ is transitive and Euclidean.
} 
P. Balbiani, D. F. Duque, and E. Lorini. The dynamics of epistemic attitudes in resourcebounded agents. Studia Logica, 2018.

A. Baltag and S. Smets. A qualitative theory of dynamic interactive belief revision. In G. Bonanno, W. van der Hoek, and M. Wooldridge, editors, Logic and the Foundations of Game and Decision Theory (LOFT 7), volume 3 of Texts in Logic and Games, pages 11-58. Amsterdam University Press, 2008.

P. Battigalli and G. Bonanno. Recent results on belief, knowledge and the epistemic foundations of game theory. Research in Economics, 53:149-225, 1999.

B. Beddor and S. Goldstein. Believing epistemic contradictions. The review of symbolic logic, 11:87-114, 2018.

P. Blackburn, M. de Rijke, and Y. Venema. Modal logic. Cambridge University Press, 2001.

C. Boutilier. Normative, subjunctive, and autoepistemic defaults: Adopting the Ramsey test. In Proceedings of the 3rd International Conference on Principles of Knowledge Representation and Reasoning (KR'92). Cambridge, MA, October 25-29, pages 685-696, 1992.

B. Chellas. Modal logic: an introduction. Cambridge University Press, 1984.

D. Dodd. Belief and certainty. Synthese, 194:4597-4621, 2017.

E. Fermé and S. O. Hansson. AGM 25 years. Journal of Philosophical Logic, 40:295-331, 2011.

E. Fermé and S. O. Hansson. Belief change: introduction and overview. Springer, 2018.

V. Fiutek. Playing with knowledge and belief. PhD thesis, University of Amsterdam, Institute for Logic, Language and Computation, 2013.

N. Friedman and J. Halpern. Modeling belief in dynamic systems. Part 1: foundations. Artificial Intelligence, 95:257-316, 1997.

E. Gettier. Is justified true belief knowledge? Analysis, 23:121-123, 1963.

M. Goldszmidt and J. Pearl. Rank-based systems: A simple approach to belief revision, belief update, and reasoning about evidence and actions. In Proceedings of the Third International Conference on Principles of Knowledge Representation and Reasoning, KR'92, pages 661-672, San Francisco, 1992. Morgan Kaufmann Publishers Inc.

J. Hintikka. Knowledge and belief: an introduction to the logic of the two notions. Cornell University Press, Cornell, 1962. 
D. Klein, O. Roy, and N. Gratzl. Knowledge, belief, normality, and introspection. Synthese, 195:4343-4372, 2018.

S. Kraus and D. Lehmann. Knowledge, belief and time. Theoretical Computer Science, 58 : 155-174, 1988.

H. E. Kyburg. Probability and the Logic of Rational Belief. Wesleyan University Press, 1961.

H. E. Kyburg. Epistemology and Inference. Univ Of Minnesota Press, 1983.

P. Lamarre and Y. Shoham. Knowledge, certainty, belief, and conditionalisation (abbreviated version). In Proceedings of the Fourth International Conference on Principles of Knowledge Representation and Reasoning, pages 415-424, San Francisco, 1994. Morgan Kaufmann Publishers Inc.

K. Lehrer and T. Paxson. Knowledge: undefeated justified true belief. Journal of Philosophy, 66:225-237, 1969.

W. Lenzen. Recent work in epistemic logic. Acta Philosophica Fennica, 30:1-219, 1978.

W. Lenzen. Epistemic logic. In I. Niiniluoto, M. Sintonen, and J. Wolenski, editors, Handbook of Epistemology, pages 963-983. Springer, 2004.

Y. Moses and Y. Shoham. Belief as defeasible knowledge. Artificial Intelligence, 64: 299-322, 1993.

E. Pacuit. Neighborhood Semantics for Modal Logic. Springer, Cham (Switzerland), 2017.

W. Spohn. Ordinal conditional functions: A dynamic theory of epistemic states. In W. L. Harper and B. Skyrms, editors, Causation in Decision, Belief Change and Statistics, volume 2, pages 105-134. D. Reidel, Dordrecht, 1988.

R. Stalnaker. On logics of knowledge and belief. Philosophical Studies, 128:169-199, 2006.

M. Swain. Epistemic defeasibility. American Philosophical Quarterly, 11:15-25, 1974.

J. van Benthem. Dynamic logic for belief revision. Journal of Applied Non-classical Logics, 17:129-155, 2007.

J. van Benthem. Logical dynamics of information and interaction. Cambridge University Press, 2011.

W. van der Hoek and J.-J. C. Meyer. Epistemic logic for AI and computer science. Cambridge University Press, Cambridge, 1995.

H. van Ditmarsch, W. van der Hoek, and B. Kooi. Dynamic epistemic logic. Springer, 2007. 
F. Voorbraak. Generalized Kripke models for epistemic logic. In Proceedings of the 4th Conference on Theoretical Aspects of Reasoning About Knowledge, TARK '92, pages 214-228, San Francisco, 1992. Morgan Kaufmann Publishers Inc.

T. Williamson. Knowledge and its limits. Oxford University Press, 2000. 